\title{
Enantiospecific Sn(II)- and Sn(IV)-Catalyzed Cycloadditions of Donor-Acceptor Cyclopropanes and Aldehydes
}

\author{
Patrick D. Pohlhaus and Jeffrey S. Johnson* \\ Department of Chemistry, University of North Carolina at Chapel Hill
}

Chapel Hill, North Carolina 27599-3290

\section{Supporting Information}

\section{Table of Contents}

\section{Experimental Section}

Materials and Methods: General. Infrared (IR) spectra were obtained using an infrared spectrometer. Proton and carbon nuclear magnetic resonance spectra $\left({ }^{1} \mathrm{H}\right.$ and $\left.{ }^{13} \mathrm{C} \mathrm{NMR}\right)$ were recorded on either a $500 \mathrm{MHz}{ }^{1} \mathrm{H}$ NMR $\left({ }^{13} \mathrm{C} \mathrm{NMR}\right.$ at $\left.125 \mathrm{MHz}\right), 400$ $\mathrm{MHz}{ }^{1} \mathrm{H}$ NMR $\left({ }^{13} \mathrm{C} \mathrm{NMR}\right.$ at $\left.100 \mathrm{MHz}\right)$, or a $300 \mathrm{MHz}{ }^{1} \mathrm{H} \mathrm{NMR}\left({ }^{13} \mathrm{C}\right.$ at $\left.75 \mathrm{MHz}\right)$ spectrometer with tetramethylsilane (TMS) or DMSO solvent resonance as the internal standard for ${ }^{1} \mathrm{H}$ NMR at $0.00 \mathrm{ppm}$ and $2.50 \mathrm{ppm}$ respectively, and $\mathrm{CDCl}_{3}$ solvent resonance as the internal standard for ${ }^{13} \mathrm{C}$ NMR at $77.16 \mathrm{ppm} .{ }^{1} \mathrm{H}$ NMR data are reported as follows: chemical shift, multiplicity $(\mathrm{s}=$ singlet, $\mathrm{d}=$ doublet, $\mathrm{t}=$ triplet, $\mathrm{q}=$ quartet, sep $=$ septet, $\mathrm{m}=$ multiplet $)$, coupling constants $(\mathrm{Hz})$, and integration. Structural assignments were made using NOESY experiments (see Appendix for spectra). Enantiomeric excesses were obtained using a Supercritical Fluid Chromatograph equipped with a UV-Vis detector using one of the following chiral HPLC columns: Chiralcel Chiralpak AD or OD column. Samples were eluted with SFC grade $\mathrm{CO}_{2}$ and the indicated percentage of $\mathrm{MeOH}$. Analytical thin layer chromatography (TLC) was performed on $0.25 \mathrm{~mm}$ silica gel 60 plates. Visualization was accomplished with UV light and aqueous ceric ammonium molybdate solution followed by heating. Purification of the reaction products was carried out by flash chromatography using silica gel 60 (32$63 \mu \mathrm{m})$. All reactions were carried out under an atmosphere of argon in flame-dried glassware with magnetic stirring. Yield refers to isolated yield of analytically pure material. Yields, diastereomeric ratios, and enantiomeric excesses are reported for a specific experiment and as a result may differ slightly from those found in the tables and equations, which are averages of at least two experiments. Methylene chloride and THF were dried by passage through a column of neutral alumina under nitrogen prior to use. DMSO was distilled from $\mathrm{CaH}_{2}$ under Ar prior to use. Solid aldehydes, 4nitrobenzaldehyde and 4-chlorobenzaldehyde were purified by sublimation prior to use. All other aldehydes were distilled from $\mathrm{CaSO}_{4}$ prior to use. The cyclopropane $(\boldsymbol{S})-\mathbf{3}$ was prepared using Davies' procedure. ${ }^{1}$ Compound 7 was prepared from the saponification of rac-3 according to Davies ${ }^{1}$ and subsequent esterification according to Corey using $d_{6^{-}}$ 
dimethyl sulfate. ${ }^{2}$ The preparation of rac-3 was accomplished using Wood's procedure. ${ }^{3}$ Unless otherwise noted, reagents were obtained from commercial sources and used without further purification.

General procedure (A) for the Lewis acid catalyzed cycloaddition. In an inert atmosphere glovebox, a flame-dried vial was charged with $0.017 \mathrm{mmol}$ of $\mathrm{Sn}(\mathrm{OTf})_{2}$ (0.050 equiv), $0.34 \mathrm{mmol}$ of the cyclopropane (1.0 equiv), and a magnetic stir bar. Outside of the glove box, the vial was placed under an argon atmosphere and charged with $0.5 \mathrm{~mL}$ of $\mathrm{CH}_{2} \mathrm{Cl}_{2}$ followed by $1.0 \mathrm{mmol}$ of the aldehyde (3.0 equiv). The reaction was stirred at room temp $\left(23{ }^{\circ} \mathrm{C}-29^{\circ} \mathrm{C}\right)$ until the disappearance of starting material was confirmed by TLC. The reaction mixture was then passed over a plug of silica with 50 $\mathrm{mL}$ of $\mathrm{Et}_{2} \mathrm{O}$, the solvent removed with a rotary evaporator, and the residue placed under vacuum $(<0.1$ torr) overnight. The product was purified by flash chromatography, eluting with the indicated solvent system to afford the pure tetrahydrofuran.

General procedure (B) for the Lewis acid catalyzed cycloaddition. In an inert atmosphere glovebox, a flame-dried vial was charged with $0.34 \mathrm{mmol}$ of the cyclopropane (1.0 equiv) and a magnetic stir bar. Outside of the glove box, the vial was

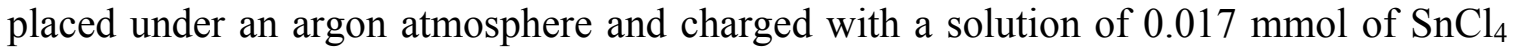
(0.050 equiv) in $0.5 \mathrm{~mL}$ of $\mathrm{CH}_{2} \mathrm{Cl}_{2}$ followed by $1.0 \mathrm{mmol}$ of the aldehyde (3.0 equiv). The reaction was stirred at room temp $\left(23{ }^{\circ} \mathrm{C}-29^{\circ} \mathrm{C}\right)$ until the disappearance of starting material was confirmed by TLC. The reaction mixture was then passed over a plug of silica with $50 \mathrm{~mL}$ of $\mathrm{Et}_{2} \mathrm{O}$, the solvent removed with a rotary evaporator, and the residue placed under vacuum $(<0.1$ torr) for a short time to remove excess aldehyde affording the pure tetrahydrofuran.
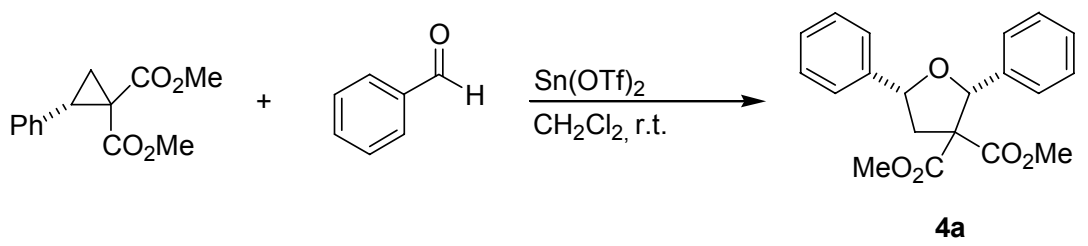

2,5-diphenyltetrahydrofuran-3,3-dicarboxylic acid dimethyl ester (4a, Table 1, entry 1). The title compound was prepared according to General Procedure A using $7.1 \mathrm{mg}$ of $\mathrm{Sn}(\mathrm{OTf})_{2}, 80.0 \mathrm{mg}$ of the cyclopropane, and $110 \mathrm{mg}$ of the aldehyde. After $2.25 \mathrm{~h}$ at room temperature and workup, ${ }^{1} \mathrm{H}$ NMR analysis of the unpurified product $(\delta 5.79 \mathrm{vs}$. $\delta$ 5.98) gave the diastereomeric ratio: $>100: 1$. The crude product was purified by flash chromatography $(7.5 \% \mathrm{EtOAc} /$ petroleum ether) to afford $117 \mathrm{mg}(100 \%)$ of the product as a colorless oil in $96 \%$ ee as determined by chiral SFC analysis (Chiralpak AD, 3.0\% $\left.\mathrm{MeOH}, 2.0 \mathrm{~mL} / \mathrm{min}, 200 \mathrm{psi}, 40^{\circ} \mathrm{C}, 254 \mathrm{~nm}, t_{\mathrm{r}-\mathrm{major}} 4.1 \mathrm{~min}, t_{\mathrm{r}-\operatorname{minor}} 5.1 \mathrm{~min}\right)$.

Analytical data for 4a: $[\alpha]_{\mathrm{D}}{ }^{25}+95.6\left(\mathrm{c}=1.01, \mathrm{CH}_{2} \mathrm{Cl}_{2}\right)$; IR (thin film, $\mathrm{cm}^{-1}$ ) 3064, 2953, 1732, 1606, 1497, 1456, 1435, 1360, 1271, 1232, 1209, 1176, 1093, 1064, 1028, 943, 902, 812, 750, 700; ${ }^{1} \mathbf{H}$ NMR $\left(300 \mathrm{MHz}, \mathrm{CDCl}_{3}\right) \delta$ 7.58-7.47 (m, 4H), 7.44$7.23(\mathrm{~m}, 6 \mathrm{H}), 5.79(\mathrm{~s}, 1 \mathrm{H}), 4.95(\mathrm{dd}, J=10.5,6.0 \mathrm{~Hz}, 1 \mathrm{H}), 3.82(\mathrm{~s}, 3 \mathrm{H}), 3.09(\mathrm{~s}, 3 \mathrm{H})$, $3.00(\mathrm{dd}, J=13.5,10.8 \mathrm{~Hz}, 1 \mathrm{H}), 2.73(\mathrm{dd}, J=13.2,6.0 \mathrm{~Hz}, 1 \mathrm{H}) ;{ }^{13} \mathbf{C} \mathbf{N M R}(100 \mathrm{MHz}$, 
$\left.\mathrm{CDCl}_{3}\right) \delta 171.5,169.3,140.0,137.8,128.6,128.22,128.17,127.9,127.1,126.6,84.6$, 80.0, 66.5, 53.0, 52.2, 42.9; TLC $\left(80 \% \mathrm{CH}_{2} \mathrm{Cl}_{2}\right.$ /petroleum ether) $\mathrm{R}_{f} 0.34$; Anal. Calcd. for $\mathrm{C}_{20} \mathrm{H}_{20} \mathrm{O}_{5}$ : C, 70.57; H, 5.92. Found: C, 70.64; H, 6.00.
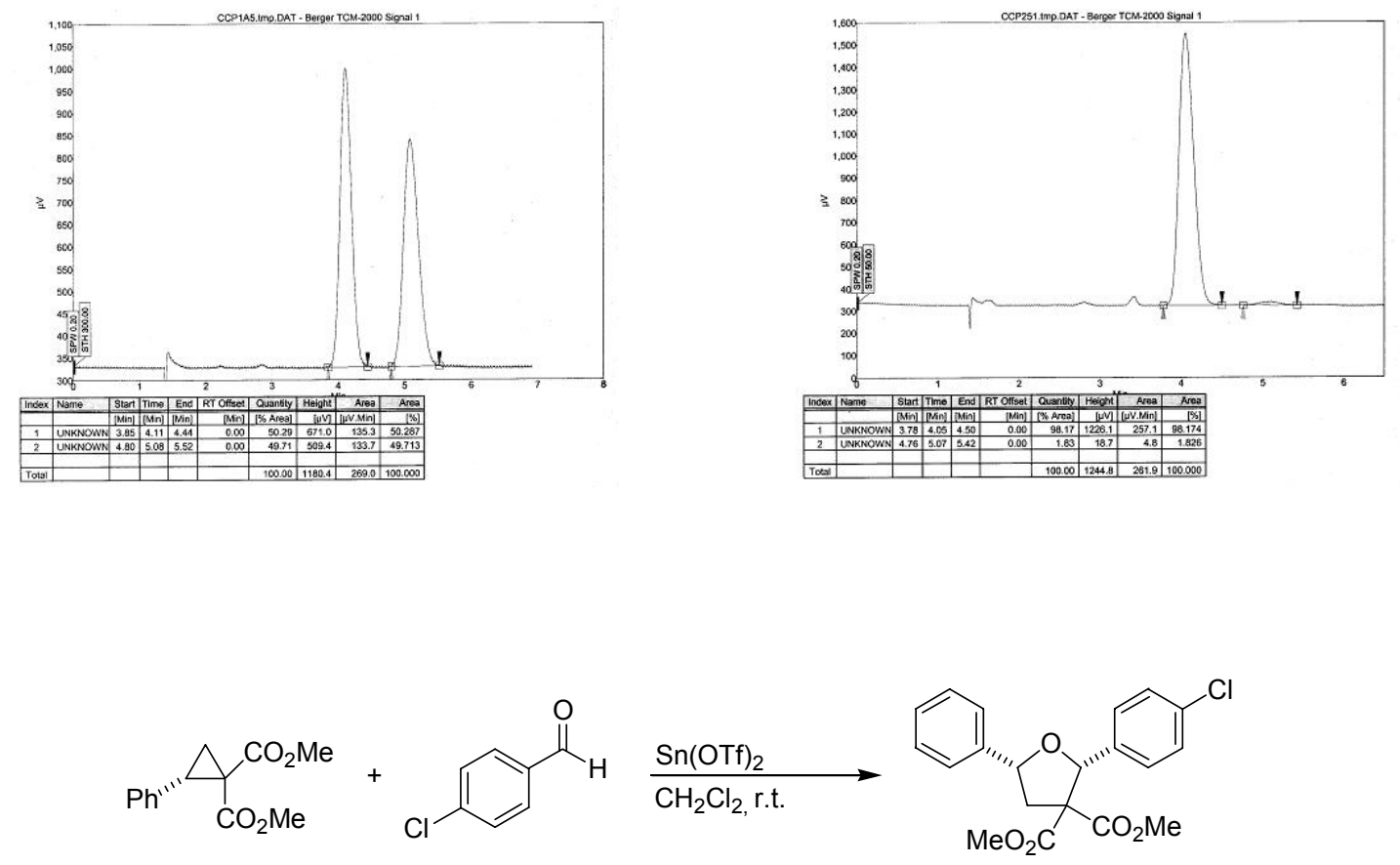

4b

(2R,5R)-2-(4-chlorophenyl)-5-phenyltetrahydrofuran-3,3-dicarboxylic acid dimethyl ester (4b, Table 1, entry 2). In an inert atmosphere glovebox, a flame-dried vial was charged with $\mathrm{Sn}(\mathrm{OTf})_{2}(7.1 \mathrm{mg}, 0.017 \mathrm{mmol}, 0.050$ equiv $)$, the cyclopropane $(80.0 \mathrm{mg}$, $0.34 \mathrm{mmol}, 1.0$ equiv), and a stir bar. Outside of the glovebox, the vial was placed under argon and charged with the aldehyde (144 mg, $1.0 \mathrm{mmol}, 3.0$ equiv) in $0.5 \mathrm{~mL}$ of $\mathrm{CH}_{2} \mathrm{Cl}_{2}$. The reaction was stirred at room temperature. After $4.75 \mathrm{~h}$ the reaction was passed over a small plug of silica with $50 \mathrm{~mL}$ of $\mathrm{Et}_{2} \mathrm{O}$, and the solvent was removed by rotary evaporation. ${ }^{1} \mathrm{H}$ NMR analysis of the unpurified product $(\delta 5.73 \mathrm{vs} . \delta 3.70)$ gave the diastereomeric ratio: $>71: 1$. The crude product was purified by flash chromatography with a $2.5 \%$ to $5 \%$ acetone/petroleum ether gradient to afford $128 \mathrm{mg}(99 \%)$ of the product as a slightly yellow oil in $95 \%$ ee as determined by chiral SFC analysis (Chiralpak OD, 3.0\% MeOH, $2.0 \mathrm{~mL} / \mathrm{min}, 200 \mathrm{psi}, 40^{\circ} \mathrm{C}, 254 \mathrm{~nm}, t_{\mathrm{r}-\text { major }} 6.8 \mathrm{~min}, t_{\mathrm{r}-\mathrm{minor}}$ $7.9 \mathrm{~min})$.

Analytical data for 4b: $[\alpha]_{\mathrm{D}}{ }^{22}+81.4\left(\mathrm{c}=1.00, \mathrm{CH}_{2} \mathrm{Cl}_{2}\right)$; IR (thin film, $\mathrm{cm}^{-1}$ ) 3034, 2953, 2883, 1732, 1599, 1491, 1435, 1385, 1348, 1271, 1232, 1209, 1198, 1176 , 1089, 1057, 1028, 1015, 941, 906, 843, 800, 760, 700; ${ }^{1} \mathbf{H}$ NMR $\left(400 \mathrm{MHz}, \mathrm{CDCl}_{3}\right) \delta$ $7.53(\mathrm{~d}, J=7.2 \mathrm{~Hz}, 2 \mathrm{H}), 7.45$ (d, $J=8.4 \mathrm{~Hz}, 2 \mathrm{H}), 7.39$ (dd, $J=7.1,7.1 \mathrm{~Hz}, 2 \mathrm{H}), 7.36-$ $7.25(\mathrm{~m}, 3 \mathrm{H}), 5.74(\mathrm{~s}, 1 \mathrm{H}), 4.94(\mathrm{dd}, J=10.4,6.0 \mathrm{~Hz}, 1 \mathrm{H}), 3.81(\mathrm{~s}, 3 \mathrm{H}), 3.16(\mathrm{~s}, 3 \mathrm{H})$, $2.97(\mathrm{dd}, J=13.6,10.8 \mathrm{~Hz}, 1 \mathrm{H}), 2.74(\mathrm{dd}, J=13.6,6.0 \mathrm{~Hz}, 1 \mathrm{H}) ;{ }^{13} \mathbf{C}$ NMR $(100 \mathrm{MHz}$, $\left.\mathrm{CDCl}_{3}\right) \delta 171.3,169.2,139.8,136.3,134.0,128.6,128.6,128.3,128.1,126.6,83.9,80.1$, 
66.3, 53.1, 52.4, 42.8; TLC (80\% $\mathrm{CH}_{2} \mathrm{Cl}_{2}$ /petroleum ether) $\mathrm{R}_{\mathrm{f}}$ 0.56; Anal. Calcd. for $\mathrm{C}_{20} \mathrm{H}_{19} \mathrm{ClO}_{5}$ : C, 64.09; H, 5.11. Found: C, 64.20; H, 5.16.
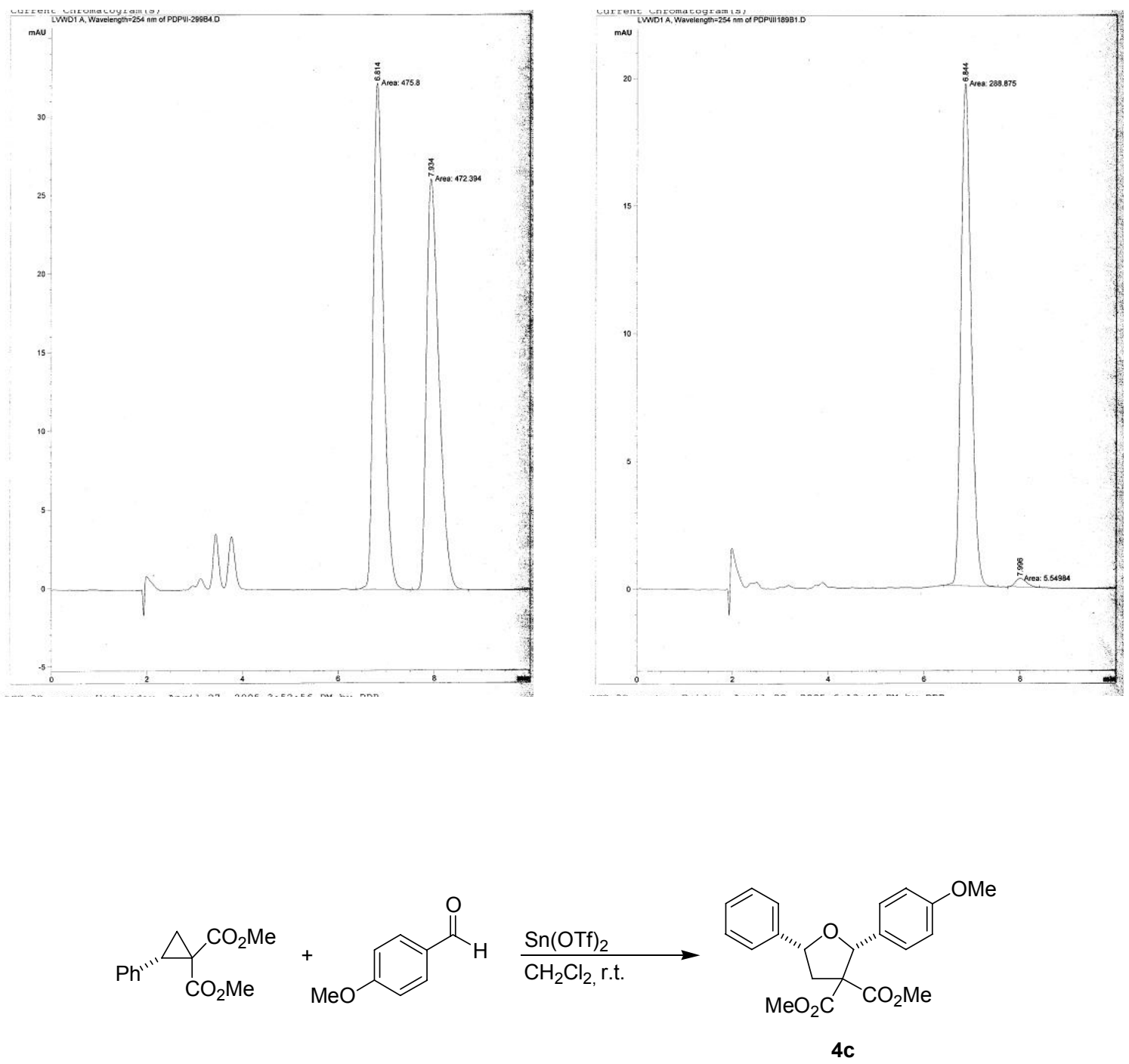

2-(4-methoxyphenyl)-5-phenyltetrahydrofuran-3,3-dicarboxylic acid dimethyl ester (4c, Table 1, entry 3). The title compound was prepared according to General Procedure A using 7.1 $\mathrm{mg}$ of $\mathrm{Sn}(\mathrm{OTf})_{2}, 80.0 \mathrm{mg}$ of the cyclopropane, and $140 \mathrm{mg}$ of the aldehyde. After $3.5 \mathrm{~h}$ at room temperature and workup, ${ }^{1} \mathrm{H}$ NMR analysis of the unpurified product $(\delta 5.75$ vs. $\delta 3.65)$ gave the diastereomeric ratio: $>71: 1$. The crude product was purified by flash chromatography with a $5 \%$ to $10 \%$ acetone/petroleum ether gradient to afford $126 \mathrm{mg}(100 \%)$ of the product as a slightly yellow oil in $99 \%$ ee as determined by chiral SFC analysis (Chiralpak AD, 3.0\% MeOH, $2.0 \mathrm{~mL} / \mathrm{min}, 200 \mathrm{psi}, 40$ $\left.{ }^{\circ} \mathrm{C}, 254 \mathrm{~nm}, t_{\mathrm{r}-\text { major }} 8.6 \mathrm{~min}, t_{\mathrm{r}-\mathrm{minor}} 9.7 \mathrm{~min}\right)$.

Analytical data for $4 \mathrm{c}:[\alpha]_{\mathrm{D}}{ }^{25}+78.4\left(\mathrm{c}=1.02, \mathrm{CH}_{2} \mathrm{Cl}_{2}\right)$; IR (thin film, $\mathrm{cm}^{-1}$ ) 3003, 2953, 2839, 1732, 1614, 1585, 1514, 1435, 1387, 1358, 1273, 1250, 1211, 1174, 
1092, 1057, 1032, 939, 904, 841, 804, 760, 700; ${ }^{\mathbf{1}} \mathbf{H}$ NMR $\left(400 \mathrm{MHz}, \mathrm{CDCl}_{3}\right) \delta 7.54(\mathrm{~d}, J$ $=7.2 \mathrm{~Hz}, 2 \mathrm{H}), 7.48-7.29(\mathrm{~m}, 5 \mathrm{H}), 6.84(\mathrm{~d}, J=8.8 \mathrm{~Hz}, 2 \mathrm{H}), 5.74(\mathrm{~s}, 1 \mathrm{H}), 4.93(\mathrm{dd}, J=$ 10.4, $6.0 \mathrm{~Hz}, 1 \mathrm{H}), 3.80(\mathrm{~s}, 3 \mathrm{H}), 3.76(\mathrm{~s}, 3 \mathrm{H}), 3.15$ (s, 3H), 2.98 (dd, $J=13.2,10.8 \mathrm{~Hz}$, $1 \mathrm{H}), 2.72(\mathrm{dd}, J=13.6,6.0 \mathrm{~Hz}, 1 \mathrm{H}) ;{ }^{13} \mathbf{C} \mathbf{N M R}\left(100 \mathrm{MHz}, \mathrm{CDCl}_{3}\right) \delta 171.5,169.4,159.6$, $140.1,129.8,128.6,128.4,128.1,126.5,113.3,84.4,79.8,66.3,55.3,52.9,52.3$, 42.8; TLC (20\% EtOAc/petroleum ether) $\mathrm{R}_{\mathrm{f}}$ 0.37; Anal. Calcd. for $\mathrm{C}_{21} \mathrm{H}_{22} \mathrm{O}_{6}$ : C, 68.10; H, 5.99. Found: C, 68.07; H, 5.94.
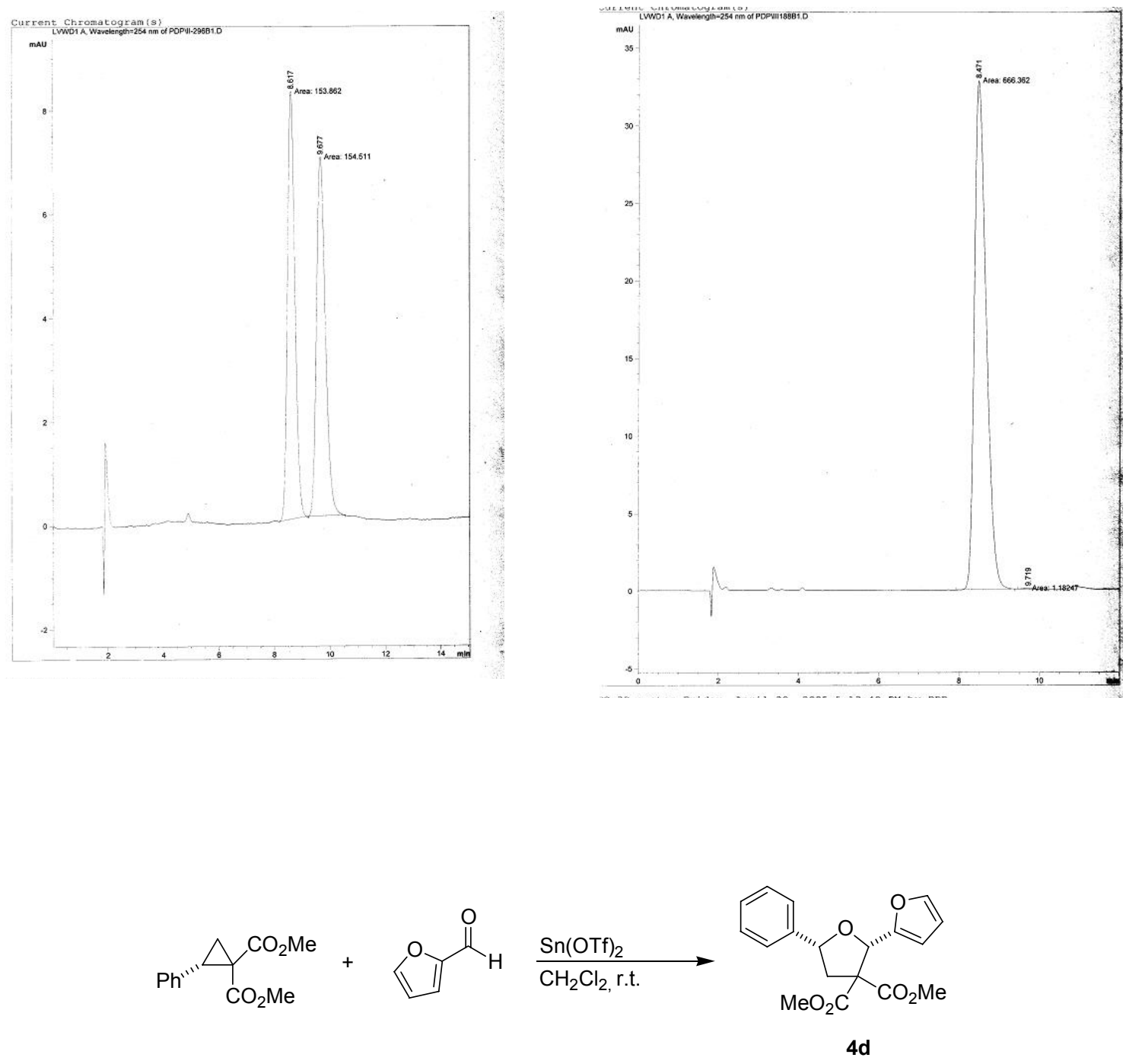

5-phenyl-1,2,3,4-tetrahydro[2,2']byfuryl-3,3-dicarboxylic acid dimethyl ester (4d, Table 1, entry 4). The title compound was prepared according to General Procedure A using $7.1 \mathrm{mg}$ of $\mathrm{Sn}(\mathrm{OTf})_{2}, 80.0 \mathrm{mg}$ of the cyclopropane, and $99 \mathrm{mg}$ of the aldehyde. After $3.25 \mathrm{~h}$ at room temperature and workup, ${ }^{1} \mathrm{H}$ NMR analysis of the unpurified product ( $\delta 4.93$ vs. $\delta 3.67$ ) gave the diastereomeric ratio: $23.3: 1$. The crude product was 
purified by flash chromatography with 5\% acetone/petroleum ether to afford $97 \mathrm{mg}$ $(86 \%)$ of the product as a colorless oil in $98 \%$ ee as determined by chiral SFC analysis (Chiralpak OD, 3.0\% MeOH, $2.0 \mathrm{~mL} / \mathrm{min}, 200 \mathrm{psi}, 40{ }^{\circ} \mathrm{C}, 254 \mathrm{~nm}$, $t_{\mathrm{r}-\text { major }} 6.5 \mathrm{~min}, t_{\mathrm{r}-\mathrm{minor}}$ $7.6 \mathrm{~min})$.

Analytical data for 4d: $[\alpha]_{\mathrm{D}}{ }^{28}+91.1\left(\mathrm{c}=1.06, \mathrm{CH}_{2} \mathrm{Cl}_{2}\right)$; IR (thin film, $\left.\mathrm{cm}^{-1}\right)$ 2954, 2359, 2343, 1736, 1497, 1450, 1435, 1333, 1273, 1232, 1209, 1173, 1151, 1093, $1051,1011,945,895,808,746,700,669 ;{ }^{1} \mathbf{H}$ NMR $\left(400 \mathrm{MHz}, \mathrm{CDCl}_{3}\right) \delta 7.47$ (d, $J=7.2$ $\mathrm{Hz}, 2 \mathrm{H}), 7.43-7.27(\mathrm{~m}, 4 \mathrm{H}), 6.38(\mathrm{~d}, J=3.1 \mathrm{~Hz}, 1 \mathrm{H}), 6.35-6.29(\mathrm{~m}, 1 \mathrm{H}), 5.85(\mathrm{~s}, 1 \mathrm{H})$, 4.93 (dd, $J=11.2,5.6 \mathrm{~Hz}, 1 \mathrm{H}), 3.83(\mathrm{~s}, 3 \mathrm{H}), 3.44(\mathrm{~s}, 3 \mathrm{H}), 3.04(\mathrm{dd}, J=13.2,11.6 \mathrm{~Hz}$, $1 \mathrm{H}), 2.72(\mathrm{dd}, J=13.6,5.6 \mathrm{~Hz}, 1 \mathrm{H}) ;{ }^{13} \mathbf{C} \mathbf{N M R}\left(100 \mathrm{MHz}, \mathrm{CDCl}_{3}\right) \delta 170.7,168.3,151.5$, $142.8,139.9,128.5,128.1,126.5,110.4,109.1,80.2,78.1,65.2,53.3,52.8,41.9$; TLC (5\% acetone/petroleum ether) $\mathrm{R}_{\mathrm{f}} 0.11$; Anal. Calcd. for $\mathrm{C}_{18} \mathrm{H}_{18} \mathrm{O}_{6}:$ C, 65.45; H, 5.49. Found: C, 65.73; H, 5.56.
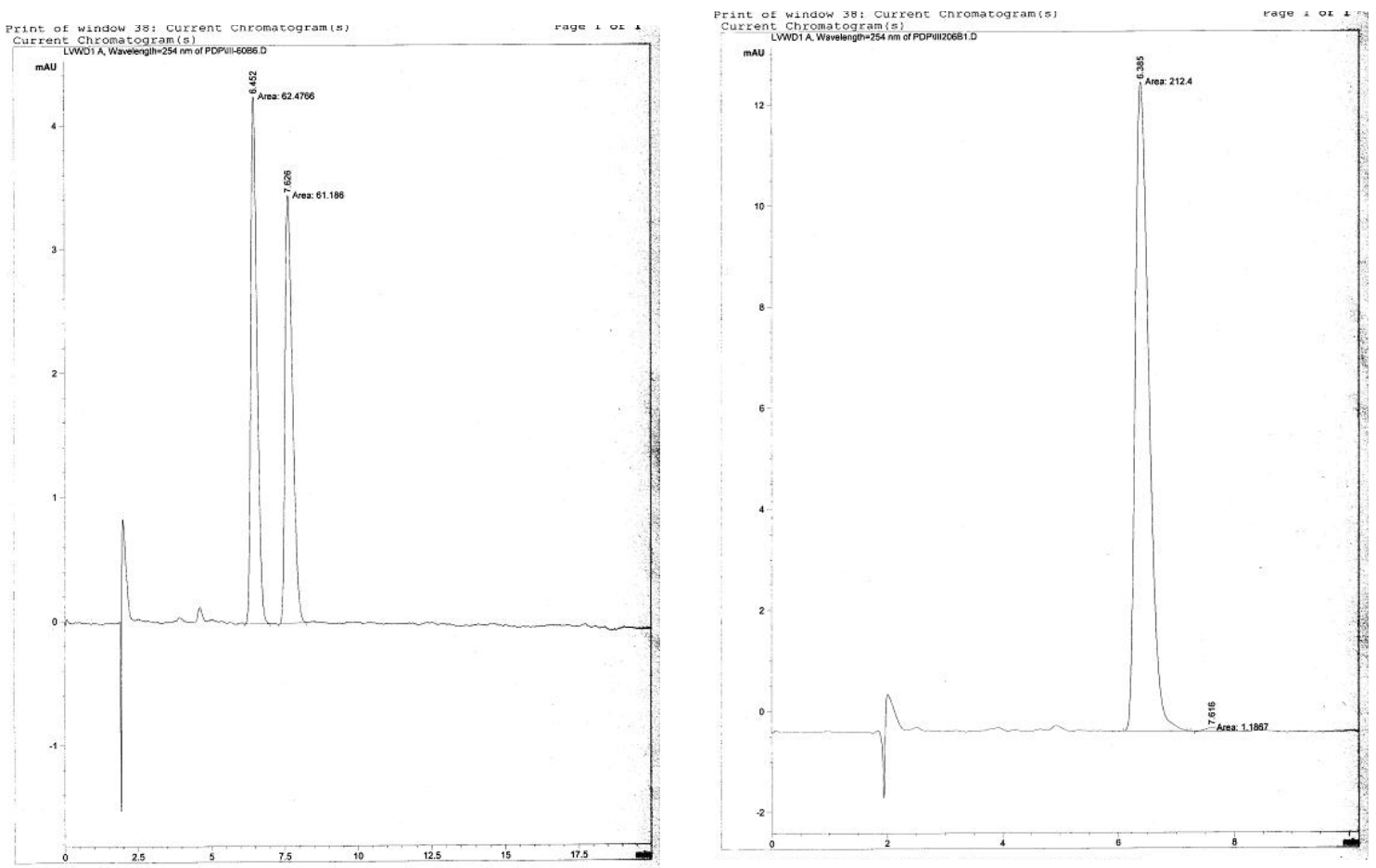


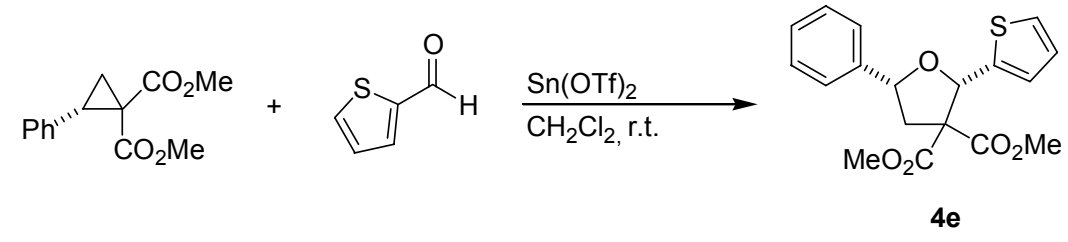

5-phenyl-2-(2-thienyl)tetrahydrofuran-3,3-dicarboxylic acid dimethyl ester (4e, Table 1, entry 5). The title compound was prepared according to General Procedure A using $7.1 \mathrm{mg}$ of $\mathrm{Sn}(\mathrm{OTf})_{2}, 80.0 \mathrm{mg}$ of the cyclopropane, and $115 \mathrm{mg}$ of the aldehyde. After $3.25 \mathrm{~h}$ at room temperature and workup, ${ }^{1} \mathrm{H}$ NMR of the unpurified product $(\delta 6.07$ vs. $\delta$ 3.68) gave the diastereomeric ratio: $>100: 1$. The crude product was purified by flash chromatography with $7.5 \%$ acetone/petroleum ether to afford $117 \mathrm{mg}(99 \%)$ of the product as a colorless oil in $99 \%$ ee as determined by chiral SFC analysis (Chiralpak AD, $\left.3.0 \% \mathrm{MeOH}, 2.0 \mathrm{~mL} / \mathrm{min}, 200 \mathrm{psi}, 40{ }^{\circ} \mathrm{C}, 254 \mathrm{~nm}, t_{\mathrm{r}-\text { major }} 12.0 \mathrm{~min}, t_{\mathrm{r}-\mathrm{minor}} 14.2 \mathrm{~min}\right)$.

Analytical data for 4e: $[\alpha]_{\mathrm{D}}{ }^{26}+123\left(\mathrm{c}=1.10, \mathrm{CH}_{2} \mathrm{Cl}_{2}\right)$; IR (thin film, $\left.\mathrm{cm}^{-1}\right) 3068$, 3034, 3007, 2953, 2359, 1732, 1498, 1439, 1367, 1321, 1273, 1236, 1213, 1198, 1174, $1092,1072,1051,1024,943,899,858,841,825,806,758,700 ;{ }^{1}$ H NMR $(400 \mathrm{MHz}$, $\left.\mathrm{CDCl}_{3}\right) \delta 7.52(\mathrm{~d}, J=7.2 \mathrm{~Hz}, 2 \mathrm{H}), 7.38(\mathrm{dd}, J=7.2,7.2 \mathrm{~Hz}, 2 \mathrm{H}), 7.34-7.28(\mathrm{~m}, 1 \mathrm{H}), 7.24$ $(\mathrm{d}, J=4.8 \mathrm{~Hz}, 1 \mathrm{H}), 7.10(\mathrm{~d}, J=3.2 \mathrm{~Hz}, 1 \mathrm{H}), 6.95(\mathrm{dd}, J=4.6,3.7 \mathrm{~Hz}, 1 \mathrm{H}), 6.06(\mathrm{~s}, 1 \mathrm{H})$, $4.94(\mathrm{dd}, J=10.8,6.0 \mathrm{~Hz}, 1 \mathrm{H}), 3.83(\mathrm{~s}, 3 \mathrm{H}), 3.32(\mathrm{~s}, 3 \mathrm{H}), 2.96$ (dd, $J=13.6,10.8 \mathrm{~Hz}$, $1 \mathrm{H}), 2.74(\mathrm{dd}, J=13.6,6.0 \mathrm{~Hz}, 1 \mathrm{H}) ;{ }^{13} \mathbf{C}$ NMR $\left(100 \mathrm{MHz}, \mathrm{CDCl}_{3}\right) \delta 171.0,168.8,141.0$, 139.7, 128.5, 128.2, 126.5, 126.4, 125.7, 125.3, 81.1, 80.1, 66.5, 53.2, 52.6, 42.1; TLC $\left(80 \% \mathrm{CH}_{2} \mathrm{Cl}_{2} /\right.$ petroleum ether) $\mathrm{R}_{\mathrm{f}}$ 0.37; Anal. Calcd. for $\mathrm{C}_{18} \mathrm{H}_{18} \mathrm{O}_{5} \mathrm{~S}: \mathrm{C}, 62.41 ; \mathrm{H}, 5.24$. Found: C, 62.20; H, 5.28.
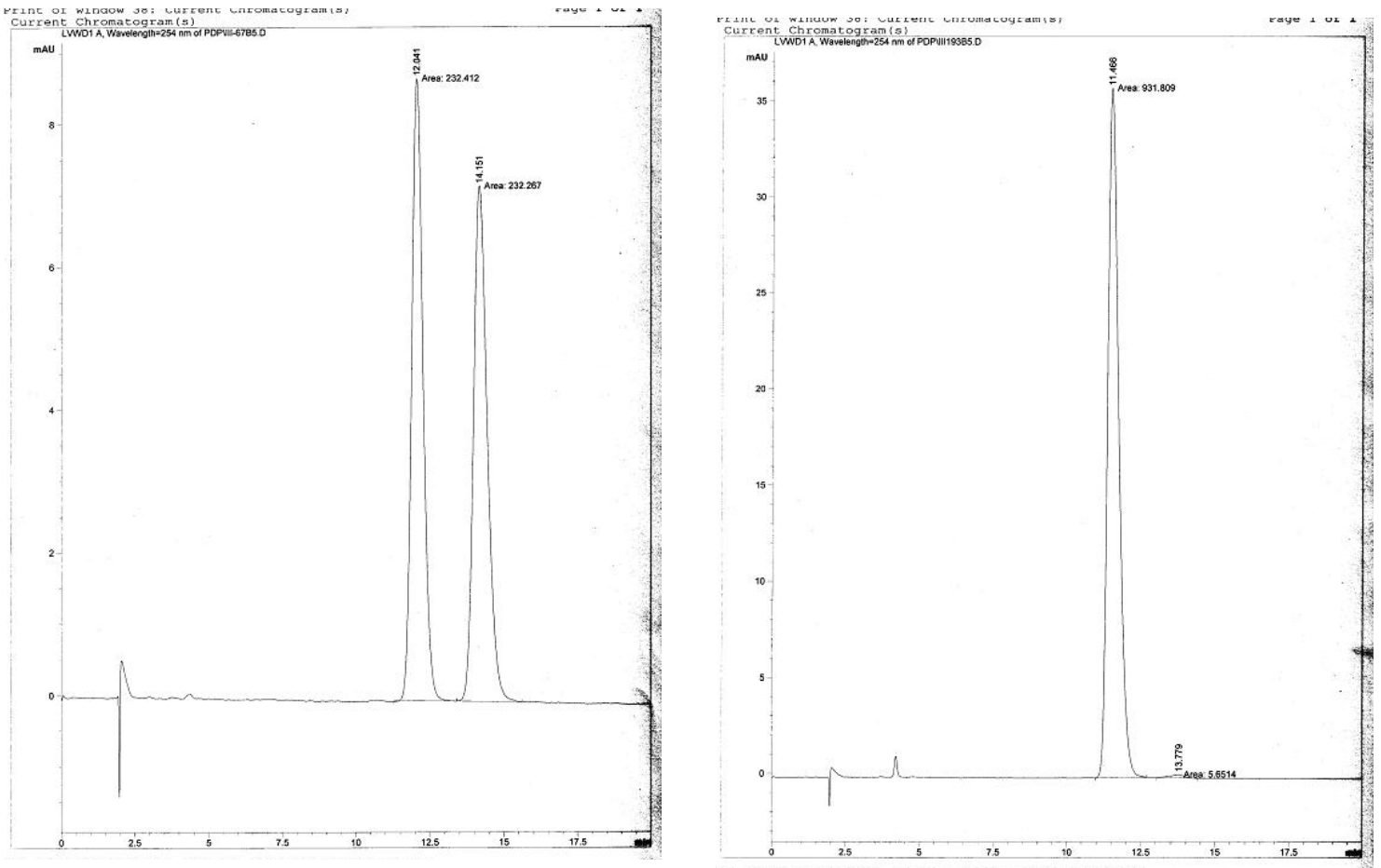


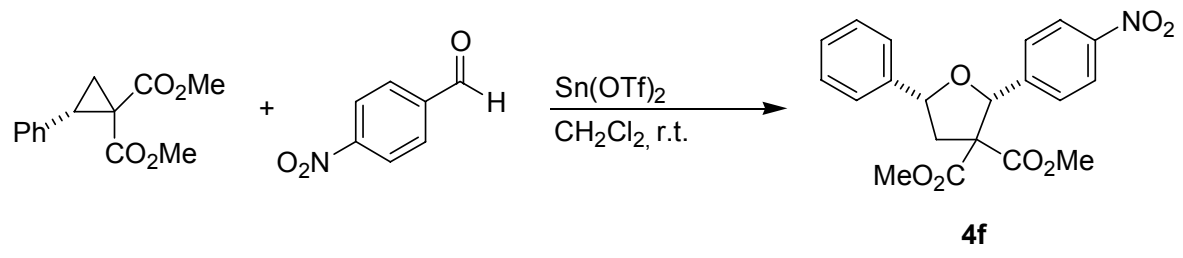

2-(4-nitrophenyl)-5-phenyltetrahydrofuran-3,3-dicarboxylic acid dimethyl ester (4f, Table 1, entry 6). In an inert atmosphere glovebox, a flame-dried vial was charged with $\mathrm{Sn}(\mathrm{OTf})_{2}(28.5 \mathrm{mg}, 0.0684 \mathrm{mmol}, 0.200$ equiv), the cyclopropane ( $80.0 \mathrm{mg}, 0.34 \mathrm{mmol}$, 1.0 equiv), and a stir bar. Outside of the glovebox, the vial was placed under argon and charged with the aldehyde (155 mg, $1.0 \mathrm{mmol}, 3.0$ equiv) in $1.0 \mathrm{~mL}$ of $\mathrm{CH}_{2} \mathrm{Cl}_{2}$. The reaction was stirred at room temperature. After $15 \mathrm{~h}$ the reaction was passed over a small plug of silica with $50 \mathrm{~mL}$ of $\mathrm{Et}_{2} \mathrm{O}$, and the solvent was removed by rotary evaporation. ${ }^{1} \mathrm{H}$ NMR analysis of the unpurified product ( $\delta 5.83$ vs. $\delta 3.82$ ) gave the diastereomeric ratio: $>52: 1$. The crude product was purified by flash chromatography with a $40 \%$ to $50 \%$ to $60 \%$ to $100 \% \mathrm{CH}_{2} \mathrm{Cl}_{2} /$ petroleum ether gradient to afford $127 \mathrm{mg}(96 \%)$ of the product as a white semi-solid in $34 \%$ ee as determined by chiral SFC analysis (Chiralpak $\mathrm{OD}, 3.0 \% \mathrm{MeOH}, 2.0 \mathrm{~mL} / \mathrm{min}, 200 \mathrm{psi}, 40{ }^{\circ} \mathrm{C}, 254 \mathrm{~nm}, t_{\mathrm{r} \text {-major }} 11.1 \mathrm{~min}, t_{\mathrm{r} \text {-minor }} 12.8 \mathrm{~min}$ ). Analytical data for $4 \mathbf{f}:[\alpha]_{\mathrm{D}}{ }^{27}+27.7\left(\mathrm{c}=1.42, \mathrm{CH}_{2} \mathrm{Cl}_{2}\right)$; IR (thin film, $\left.\mathrm{cm}^{-1}\right) 2954$, $1732,1606,1524,1495,1435,1387,1348,1313,1273,1232,1209,1176,1109,1092$, $1059,1030,1016,941,862,845,806,762,746,698 ;{ }^{1} \mathbf{H}$ NMR $\left(400 \mathrm{MHz}, \mathrm{CDCl}_{3}\right) \delta 8.18$ $(\mathrm{d}, J=8.8 \mathrm{~Hz}, 2 \mathrm{H}), 7.72(\mathrm{~d}, J=8.4 \mathrm{~Hz}, 2 \mathrm{H}), 7.55(\mathrm{~d}, J=7.2 \mathrm{~Hz}, 2 \mathrm{H}), 7.42(\mathrm{dd}, J=7.2$, $7.2 \mathrm{~Hz}, 2 \mathrm{H}), 7.39-7.31(\mathrm{~m}, 1 \mathrm{H}), 5.82(\mathrm{~s}, 1 \mathrm{H}), 4.99(\mathrm{dd}, J=10.0,6.8 \mathrm{~Hz}, 1 \mathrm{H}), 3.85$ (s, $3 \mathrm{H}), 3.15(\mathrm{~s}, 3 \mathrm{H}), 3.00(\mathrm{dd}, J=13.2,10.8 \mathrm{~Hz}, 1 \mathrm{H}), 2.81(\mathrm{dd}, J=13.2,6.4 \mathrm{~Hz}, 1 \mathrm{H}) ;{ }^{13} \mathbf{C}$ NMR $\left(100 \mathrm{MHz}, \mathrm{CDCl}_{3}\right) \delta 171.0,169.0,147.8,145.0,139.3,128.7,128.5,128.1,126.6$, 123.0, 83.5, 80.4, 66.4, 53.3, 52.5, 42.9; TLC (20\% EtOAc/petroleum ether) $\mathrm{R}_{\mathrm{f}} 0.36$; Anal. Calcd. for $\mathrm{C}_{20} \mathrm{H}_{19} \mathrm{NO}_{7}$ : C, 62.33; H, 4.97; N, 3.63. Found: C, 62.05; H, 4.96; N, 3.65 . 

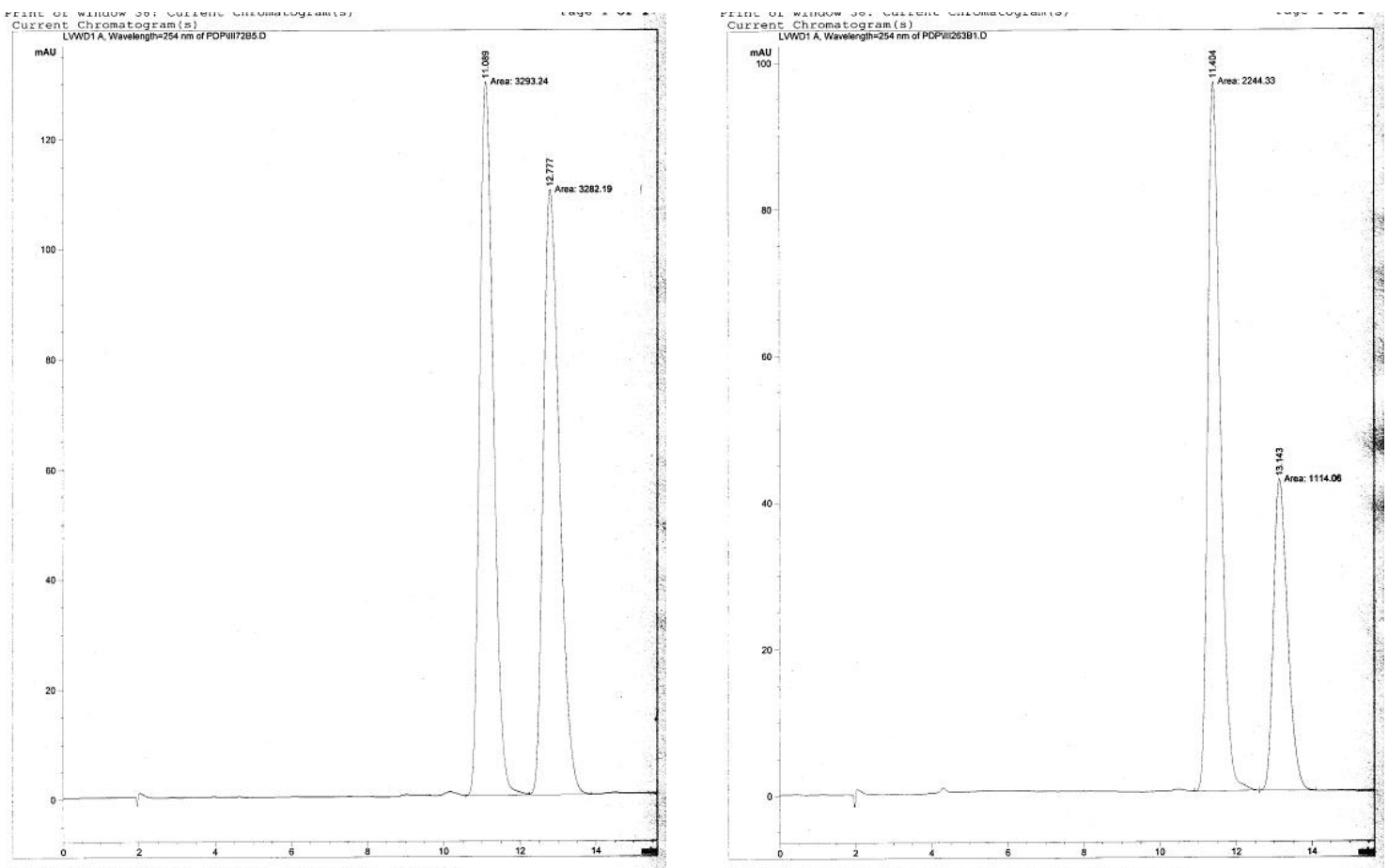

$\underline{\text { After } 45 \text { minute reaction and quench }}$

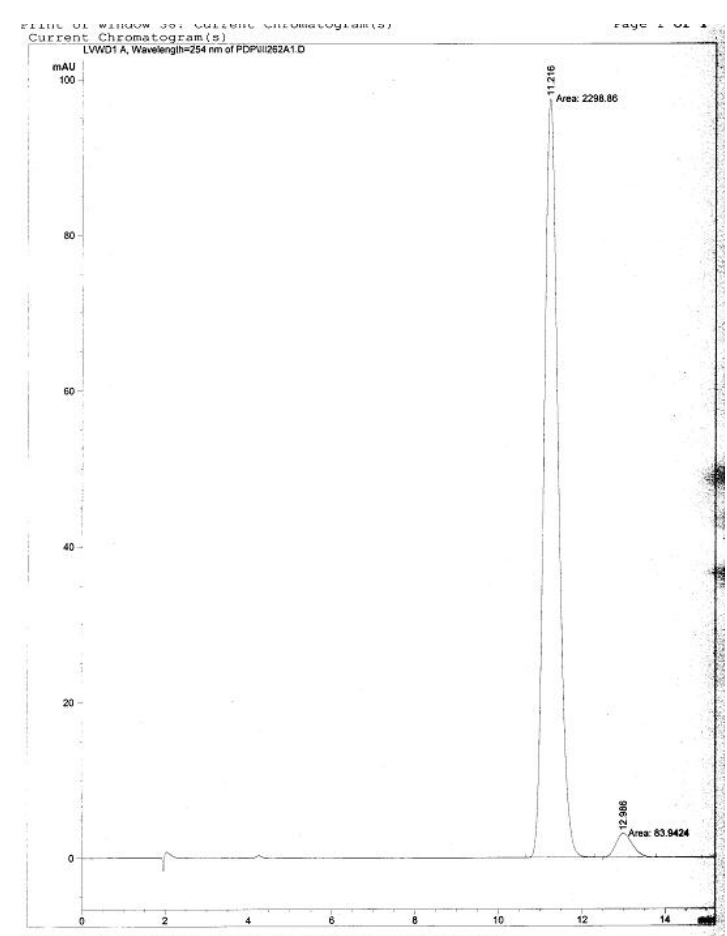




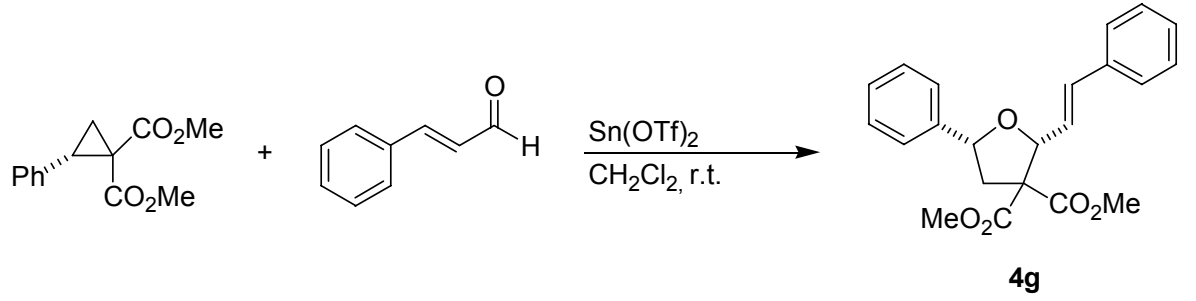

5-phenyl-2-styryltetrahydrofuran-3,3-dicarboxylic acid dimethyl ester (4g, Table 1, entry 7). The title compound was prepared according to General Procedure A using 7.1 $\mathrm{mg}$ of $\mathrm{Sn}(\mathrm{OTf})_{2}, 80.0 \mathrm{mg}$ of the cyclopropane, and $137 \mathrm{mg}$ of the aldehyde. After $3.75 \mathrm{~h}$ at room temperature and workup, ${ }^{1} \mathrm{H}$ NMR of the unpurified product ( $\delta 4.91$ vs. $\delta 3.69$ ) gave the diastereomeric ratio: 17.5:1. The isomers were separated and purified by flash chromatography with a $3 \%$ to $5 \%$ acetone/petroleum ether gradient to afford $126 \mathrm{mg}$ $(100 \%)$ of the products as yellow oils with the major diastereomer in $99 \%$ ee as determined by chiral SFC analysis (Chiralpak OD, 3.0\% MeOH, $2.0 \mathrm{~mL} / \mathrm{min}, 200 \mathrm{psi}, 40$ ${ }^{\circ} \mathrm{C}, 254 \mathrm{~nm}, t_{\mathrm{r}-\text { major }} 9.8 \mathrm{~min}, t_{\mathrm{r}-\text { minor }} 11.7 \mathrm{~min}$ ).

Analytical data for $4 \mathrm{~g}$ : $[\alpha]_{\mathrm{D}}{ }^{25}+105.6\left(\mathrm{c}=1.00, \mathrm{CH}_{2} \mathrm{Cl}_{2}\right)$; IR (thin film, $\mathrm{cm}^{-1}$ ) 3084, 3057, 3030, 3003, 2953, 2927, 2848, 1732, 1657, 1601, 1578, 1497, 1450, 1435, 1387, 1354, 1335, 1273, 1225, 1198, 1176, 1140, 1090, 1086, 1043, 1005, 970, 945, 914, 845, 818, 796, 760, 748, 696; ${ }^{1} \mathbf{H}$ NMR (500 MHz, $\left.\mathrm{CDCl}_{3}\right) \delta 7.50-7.45(\mathrm{~m}, 2 \mathrm{H}), 7.40-$ $7.20(\mathrm{~m}, 8 \mathrm{H}), 6.80(\mathrm{~d}, J=16.0 \mathrm{~Hz}, 1 \mathrm{H}), 6.23(\mathrm{dd}, J=16.0,7.0 \mathrm{~Hz}, 1 \mathrm{H}), 5.28(\mathrm{dd}, J=7.0$, $1.0 \mathrm{~Hz}, 1 \mathrm{H}), 4.91(\mathrm{dd}, J=10.5,6.5 \mathrm{~Hz}, 1 \mathrm{H}), 3.84(\mathrm{~s}, 3 \mathrm{H}), 3.62(\mathrm{~s}, 3 \mathrm{H}), 2.85(\mathrm{dd}, J=13.5$, $10.0 \mathrm{~Hz}, 1 \mathrm{H}), 2.77(\mathrm{dd}, J=13.5,6.5 \mathrm{~Hz}, 1 \mathrm{H}) ;{ }^{13} \mathbf{C} \mathbf{N M R}\left(125 \mathrm{MHz}, \mathrm{CDCl}_{3}\right) \delta 171.0$, $169.4,140.2$, 136.5, 133.1, 128.7, 128.6, 128.2, 128.0, 126.8, 126.6, 125.0, 83.2, 80.2, 65.3, 53.2, 52.9, 42.3; TLC (80\% $\mathrm{CH}_{2} \mathrm{Cl}_{2}$ /petroleum ether) $\mathrm{R}_{\mathrm{f}}$ 0.38; Anal. Calcd. for $\mathrm{C}_{22} \mathrm{H}_{22} \mathrm{O}_{5}$ : C, 72.12; H, 6.05. Found: C, 72.25; H, 6.20. 

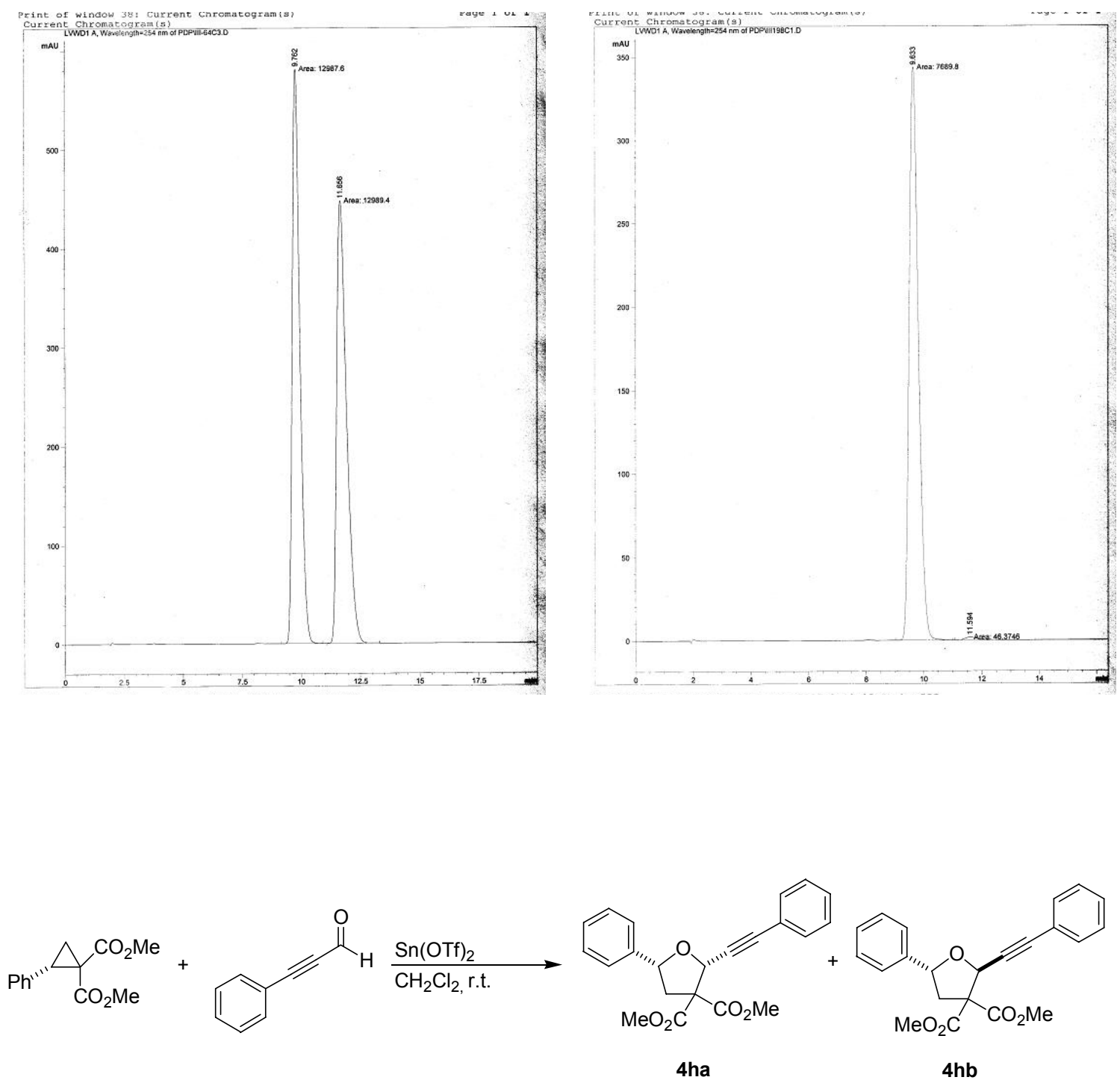

cis-5-phenyl-2-phenylethynyltetrahydrofuran-3,3-dicarboxylic acid dimethyl ester (4ha, Table 1, entry 8). In an inert atmosphere glovebox, a flame-dried vial was charged with $\mathrm{Sn}(\mathrm{OTf})_{2}(14.2 \mathrm{mg}, 0.034 \mathrm{mmol}, 0.10$ equiv), the cyclopropane $(80.0 \mathrm{mg}$, $0.34 \mathrm{mmol}, 1.0$ equiv), and a stir bar. Outside of the glovebox, the vial was placed under argon and charged with $0.5 \mathrm{~mL}$ of $\mathrm{CH}_{2} \mathrm{Cl}_{2}$ and the aldehyde $(133 \mathrm{mg}, 1.0 \mathrm{mmol}, 3.0$ equiv). The reaction was stirred at room temperature. After $6 \mathrm{~h}$ the reaction was passed over a small plug of silica with $50 \mathrm{~mL}$ of $\mathrm{Et}_{2} \mathrm{O}$, the solvent removed by rotary evaporation, and the residue placed under vacuum $(<0.1$ torr $)$ overnight. ${ }^{1} \mathrm{H} \mathrm{NMR}$ analysis of the unpurified product ( $\delta 5.67$ vs. $\delta 5.93)$ gave the diastereomeric ratio: 1.6:1. The isomers were separated and purified by flash chromatography with a $7.5 \%$ to $10 \%$ acetone/petroleum ether gradient to afford $106 \mathrm{mg}(85 \%)$ of the products as yellow oils with the major diastereomer in $88 \%$ ee as determined by chiral SFC analysis (Chiralpak $\left.\mathrm{OD}, 3.0 \% \mathrm{MeOH}, 2.0 \mathrm{~mL} / \mathrm{min}, 200 \mathrm{psi}, 40{ }^{\circ} \mathrm{C}, 254 \mathrm{~nm}, t_{\mathrm{r}-\text { major }} 9.4, t_{\mathrm{r}-\text { minor }} 11.7 \mathrm{~min}\right)$.

Analytical data for 4ha: $[\alpha]_{\mathrm{D}}{ }^{26}+82.4\left(\mathrm{c}=0.690, \mathrm{CH}_{2} \mathrm{Cl}_{2}\right)$; IR (thin film, $\left.\mathrm{cm}^{-1}\right)$ 3064,3032 , 3005, 2953, 2231, 1738, 1599, 1574, 1491, 1435, 1383, 1354, 1327, 1273, 
1228, 1196, 1173, 1092, 1070 1049, 1028, 1001, 989, 955, 914, 887, 843, 804, 758, 692;

${ }^{1} \mathbf{H}$ NMR $\left(400 \mathrm{MHz}, \mathrm{CDCl}_{3}\right) \delta$ 7.60-7.23 (m, 10H), $5.66(\mathrm{~s}, 1 \mathrm{H}), 4.96(\mathrm{dd}, J=10.8,6.0$ $\mathrm{Hz}, 1 \mathrm{H}), 3.86$ (s, 3H), 3.77 (s, 3H), 2.93 (dd, $J=13.2,10.8 \mathrm{~Hz}, 1 \mathrm{H}), 2.82$ (dd, $J=13.2$, $5.6 \mathrm{~Hz}, 1 \mathrm{H}) ;{ }^{13} \mathrm{C}$ NMR $\left(100 \mathrm{MHz}, \mathrm{CDCl}_{3}\right) \delta 169.8,168.0,140.3,131.8,128.8,128.6$, $128.4,128.1,126.5,122.2,87.8,85.2,81.1,73.0,66.6,53.5,53.1,41.5$; TLC $(10 \%$ acetone/petroleum ether) $\mathrm{R}_{\mathrm{f}} 0.17$; Anal. Calcd. for $\mathrm{C}_{22} \mathrm{H}_{20} \mathrm{O}_{5}: \mathrm{C}, 72.51 ; \mathrm{H}, 5.53$. Found: C, 72.79; H, 5.48 .

Analytical data for trans-5-phenyl-2-phenylethynyltetrahydrofuran-3,3dicarboxylic acid dimethyl ester (4hb, Table 1, entry 8): IR (thin film, $\mathrm{cm}^{-1}$ ) 3064, 3034, 3003, 2953, 2850, 2227, 1740, 1599, 1491, 1435, 1331, 1269, 1228, 1198, 1171, 1109, 1049, 1001, 987, 970, 939, 916, 889, 758, 692; ${ }^{1} \mathbf{H}$ NMR (400 MHz, CDCl 3 ) $\delta 7.50-7.24(\mathrm{~m}, 10 \mathrm{H}), 5.91(\mathrm{~s}, 1 \mathrm{H}), 5.41(\mathrm{dd}, J=7.6,7.6 \mathrm{~Hz}, 1 \mathrm{H}), 3.79(\mathrm{~s}, 3 \mathrm{H}), 3.74(\mathrm{~s}$, $3 \mathrm{H}), 3.40(\mathrm{dd}, J=13.2,7.2 \mathrm{~Hz}, 1 \mathrm{H}), 2.23(\mathrm{dd}, J=13.6,8.4 \mathrm{~Hz}, 1 \mathrm{H}) ;{ }^{13} \mathbf{C}$ NMR $(100$ $\left.\mathrm{MHz}_{,} \mathrm{CDCl}_{3}\right) \delta 170.1,168.4,140.5,132.0,128.9,128.6,128.4,128.0,126.0,122.2$, $87.8,84.6,80.4,73.3,66.6,53.3$ (two overlapping signals), 41.5; TLC (10\% acetone/petroleum ether) $\mathrm{R}_{\mathrm{f}}$ 0.24; Anal. Calcd. for $\mathrm{C}_{22} \mathrm{H}_{20} \mathrm{O}_{5}: \mathrm{C}, 72.51 ; \mathrm{H}, 5.53$. Found: C, 72.63; H, 5.49.
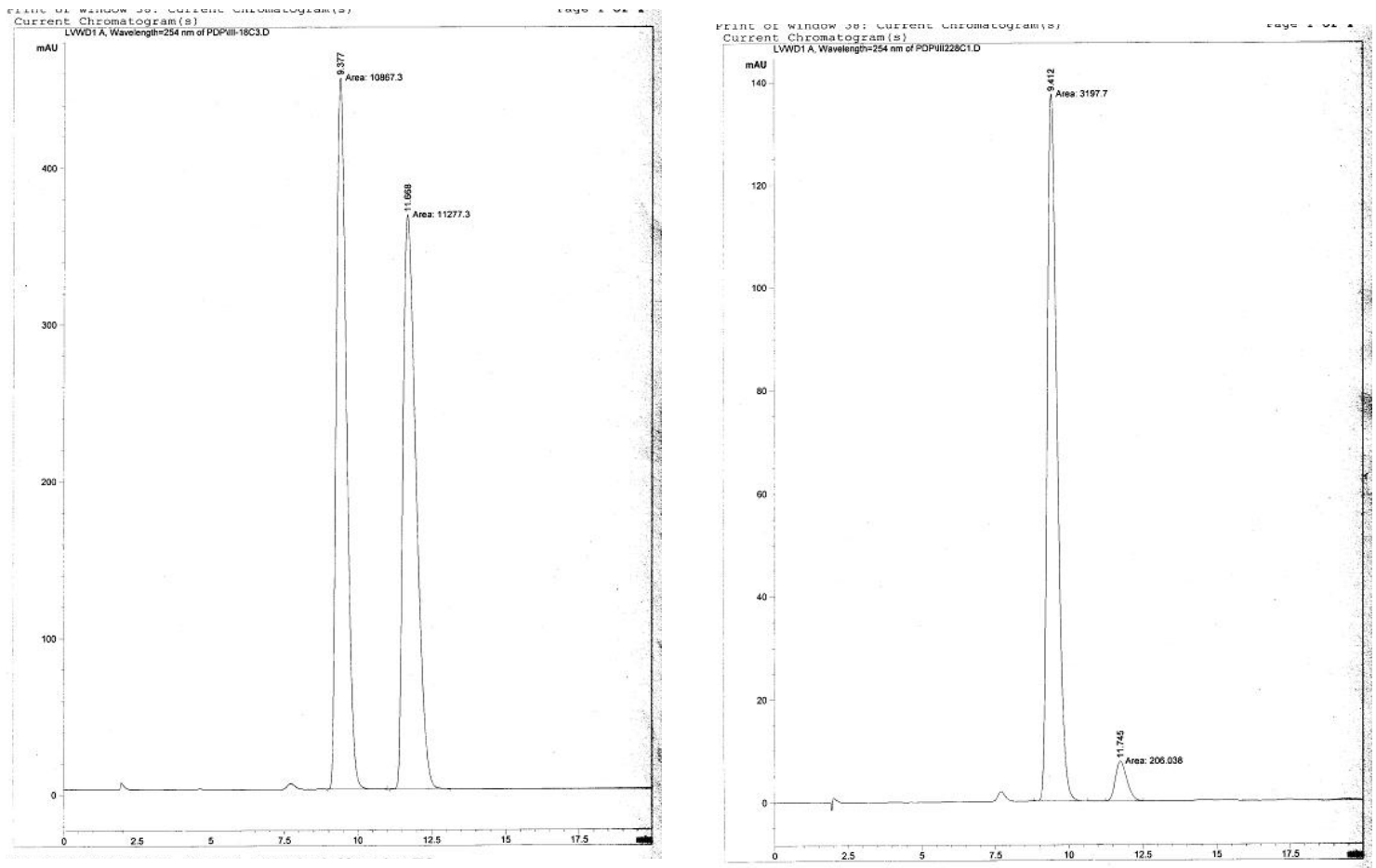
After 45 minute reaction and quench
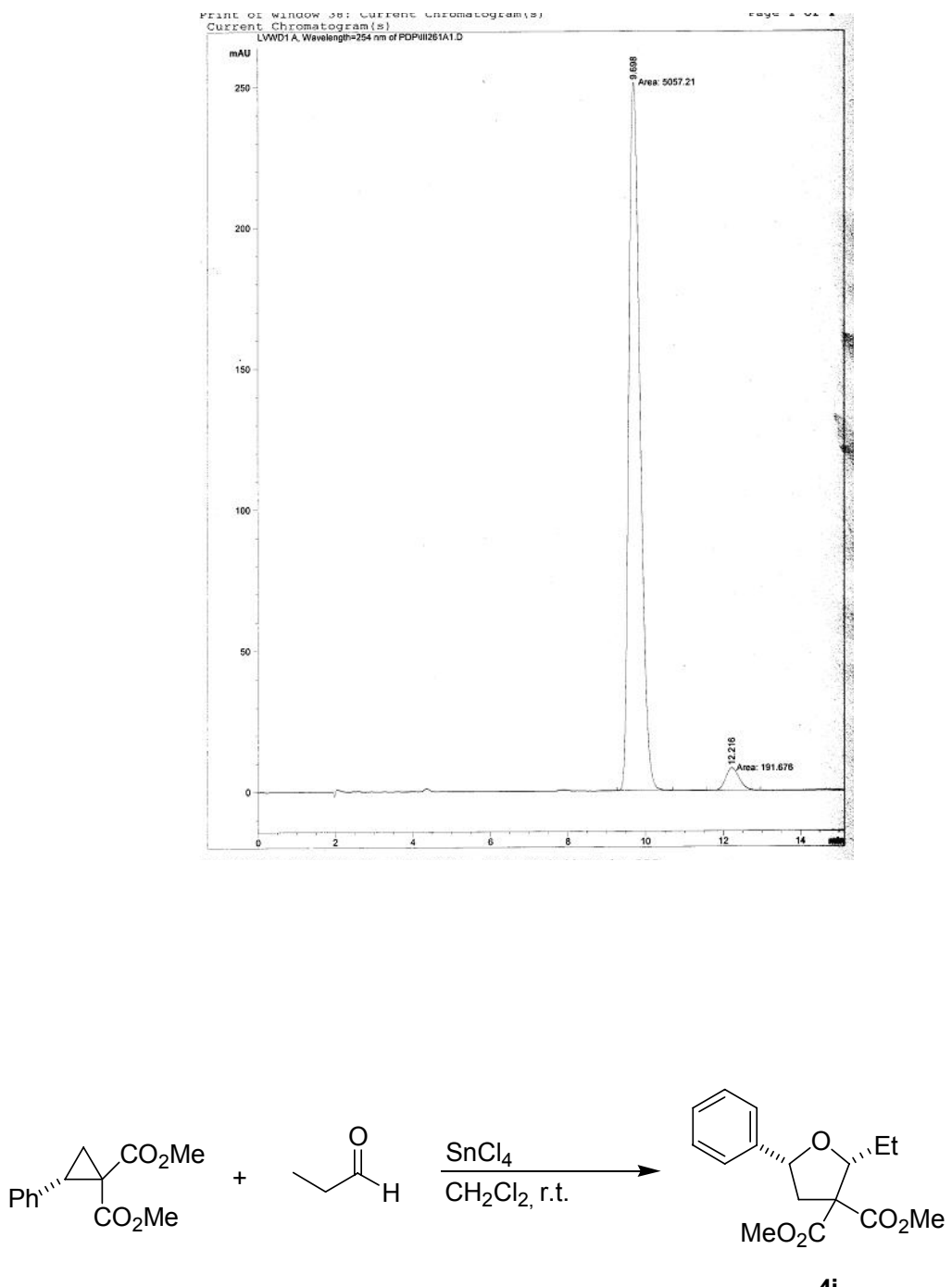

$4 \mathbf{i}$

2-Ethyl-5-phenyl-dihydro-furan-3,3-dicarboxylic acid dimethyl ester (4i, Table 1, entry 9). The title compound was prepared according to General Procedure B using $4.45 \mathrm{mg}$ of $\mathrm{SnCl}_{4}, 80.0 \mathrm{mg}$ of the cyclopropane, and $60 \mathrm{mg}$ of the aldehyde. After $1.75 \mathrm{~h}$ at room temperature and workup, ${ }^{1} \mathrm{H}$ NMR analysis of the product ( $\delta 4.79$ vs. $\delta 3.68$ ) gave the diastereomeric ratio: $>37: 1$. The pure product was obtained as $100 \mathrm{mg}(100 \%)$ of a slightly yellow oil. This product was converted to 10, which was determined to be $96 \%$ ee by chiral SFC analysis (Chiralpak AD, 10.0\% MeOH, $2.0 \mathrm{~mL} / \mathrm{min}, 200 \mathrm{psi}, 40$ ${ }^{\circ} \mathrm{C}, 225 \mathrm{~nm}, t_{\mathrm{r}-\text { major }} 9.2 \mathrm{~min}, t_{\mathrm{r}-\text { minor }} 6.9 \mathrm{~min}$ ).

Analytical data for 4i: $[\alpha]_{\mathrm{D}}{ }^{27}+101.9\left(\mathrm{c}=1.02, \mathrm{CH}_{2} \mathrm{Cl}_{2}\right)$; IR (thin film, $\left.\mathrm{cm}^{-1}\right)$ 3032, 2954, 2879, 1734, 1497, 1456, 1435, 1269, 1232, 1198, 1176, 1132, 1093, 1068, 1028, 993, 758, 700; ${ }^{1} \mathbf{H}$ NMR $\left(500 \mathrm{MHz}, \mathrm{CDCl}_{3}\right) \delta 7.42(\mathrm{~d}, J=7.5 \mathrm{~Hz}, 2 \mathrm{H}), 7.34$ (dd, $J$ $=7.0,7.0 \mathrm{~Hz}, 2 \mathrm{H}), 7.27(\mathrm{t}, J=7.5 \mathrm{~Hz}, 1 \mathrm{H}), 4.79(\mathrm{dd}, J=8.5,8.5 \mathrm{~Hz}, 1 \mathrm{H}), 4.43(\mathrm{dd}, J=$ 
10, 3.0 Hz, 1H), 3.79 (s, 3H), $3.73(\mathrm{~s}, 3 \mathrm{H}), 2.71(\mathrm{~d}, J=8.5 \mathrm{~Hz}, 2 \mathrm{H}), 1.78-1.67(\mathrm{~m}, 1 \mathrm{H})$, $1.59-1.45(\mathrm{~m}, 1 \mathrm{H}), 1.09(\mathrm{t}, J=7.5 \mathrm{~Hz}, 3 \mathrm{H}) ;{ }^{13} \mathbf{C} \mathbf{N M R}\left(125 \mathrm{MHz}, \mathrm{CDCl}_{3}\right) \delta 171.2,170.1$, $140.6,128.4,127.9,126.4,84.5,79.6,63.8,52.9,52.6,42.8,25.0,11.4$; TLC (10\% EtOAc/petroleum ether) $\mathrm{R}_{f} 0.23$; Anal. Calcd. for $\mathrm{C}_{16} \mathrm{H}_{20} \mathrm{O}_{5}: \mathrm{C}, 65.74 ; \mathrm{H}, 6.90$. Found: $\mathrm{C}$, 65.66; H, 6.88.
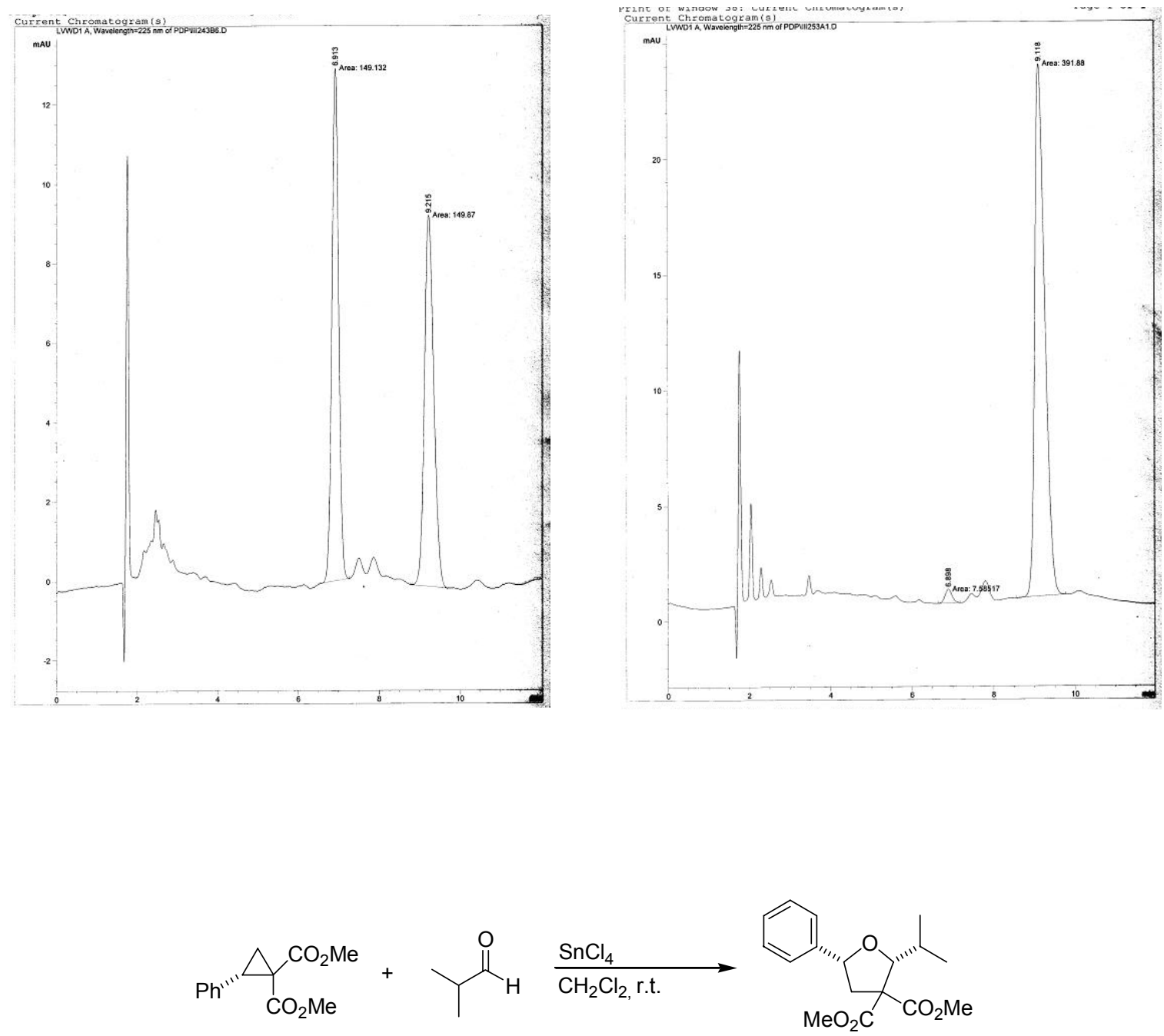

$4 \mathbf{j}$

2-Isopropyl-5-phenyl-dihydro-furan-3,3-dicarboxylic acid dimethyl ester $(4 \mathbf{j}$, Table 1, entry 10). The title compound was prepared according to General Procedure B using $4.45 \mathrm{mg}$ of $\mathrm{SnCl}_{4}, 80.0 \mathrm{mg}$ of the cyclopropane, and $74 \mathrm{mg}$ of the aldehyde. After $2.5 \mathrm{~h}$ at room temperature and workup, ${ }^{1} \mathrm{H} \mathrm{NMR}$ analysis of the product ( $\delta 4.76$ vs. $\delta 3.84$ ) gave the diastereomeric ratio: $>100: 1$. The pure product was obtained as $100 \mathrm{mg}(96 \%)$ of a colorless oil. This product was converted to 11, which was determined to be $95 \%$ ee 
by chiral SFC analysis (Chiralpak AD, 10.0\% MeOH, $2.0 \mathrm{~mL} / \mathrm{min}, 200 \mathrm{psi}, 40{ }^{\circ} \mathrm{C}, 225$ $\left.\mathrm{nm}, t_{\mathrm{r}-\mathrm{major}} 7.9 \mathrm{~min}, t_{\mathrm{r}-\mathrm{minor}} 6.7 \mathrm{~min}\right)$.

Analytical data for 4j: $[\alpha]_{\mathrm{D}}{ }^{26}+94.4\left(\mathrm{c}=1.01, \mathrm{CH}_{2} \mathrm{Cl}_{2}\right)$; IR (thin film, $\left.\mathrm{cm}^{-1}\right) 3066$, 3030, 2954, 2873, 1732, 1498, 1450, 1435, 1385, 1369, 1348, 1313, 1269, 1232, 1198 , 1176, 1099, 1061, 1022, 760, 700; ${ }^{1} \mathbf{H}$ NMR $\left(500 \mathrm{MHz}, \mathrm{CDCl}_{3}\right) \delta 7.41(\mathrm{~d}, J=7.0 \mathrm{~Hz}$, 2H), $7.34(\mathrm{dd}, J=7.5,7.5 \mathrm{~Hz}, 2 \mathrm{H}), 7.27$ (t, $J=7.5 \mathrm{~Hz}, 1 \mathrm{H}), 4.76(\mathrm{dd}, J=9.5,7.5 \mathrm{~Hz}$, $1 \mathrm{H}), 4.31(\mathrm{~d}, J=7.0 \mathrm{~Hz}, 1 \mathrm{H}), 3.79(\mathrm{~s}, 3 \mathrm{H}), 3.74(\mathrm{~s}, 3 \mathrm{H}), 2.77-2.66(\mathrm{~m}, 2 \mathrm{H}), 2.03-1.93(\mathrm{~m}$, $1 \mathrm{H}), 1.03(\mathrm{~d}, J=7.0 \mathrm{~Hz}, 3 \mathrm{H}), 1.02(\mathrm{~d}, J=6.5 \mathrm{~Hz}, 3 \mathrm{H}) ;{ }^{13} \mathbf{C ~ N M R}\left(125 \mathrm{MHz}, \mathrm{CDCl}_{3}\right) \delta$ $171.7,170.5,140.6,128.4,127.8,126.4,88.4,78.9,63.2,52.9,52.6,44.4,30.1,20.6$, 19.1; TLC (20\% EtOAc/petroleum ether) $\mathrm{R}_{f} 0.48$; Anal. Calcd. for $\mathrm{C}_{17} \mathrm{H}_{22} \mathrm{O}_{5}$ : C, 66.65; H, 7.24. Found: C, 66.85; H, 7.11.
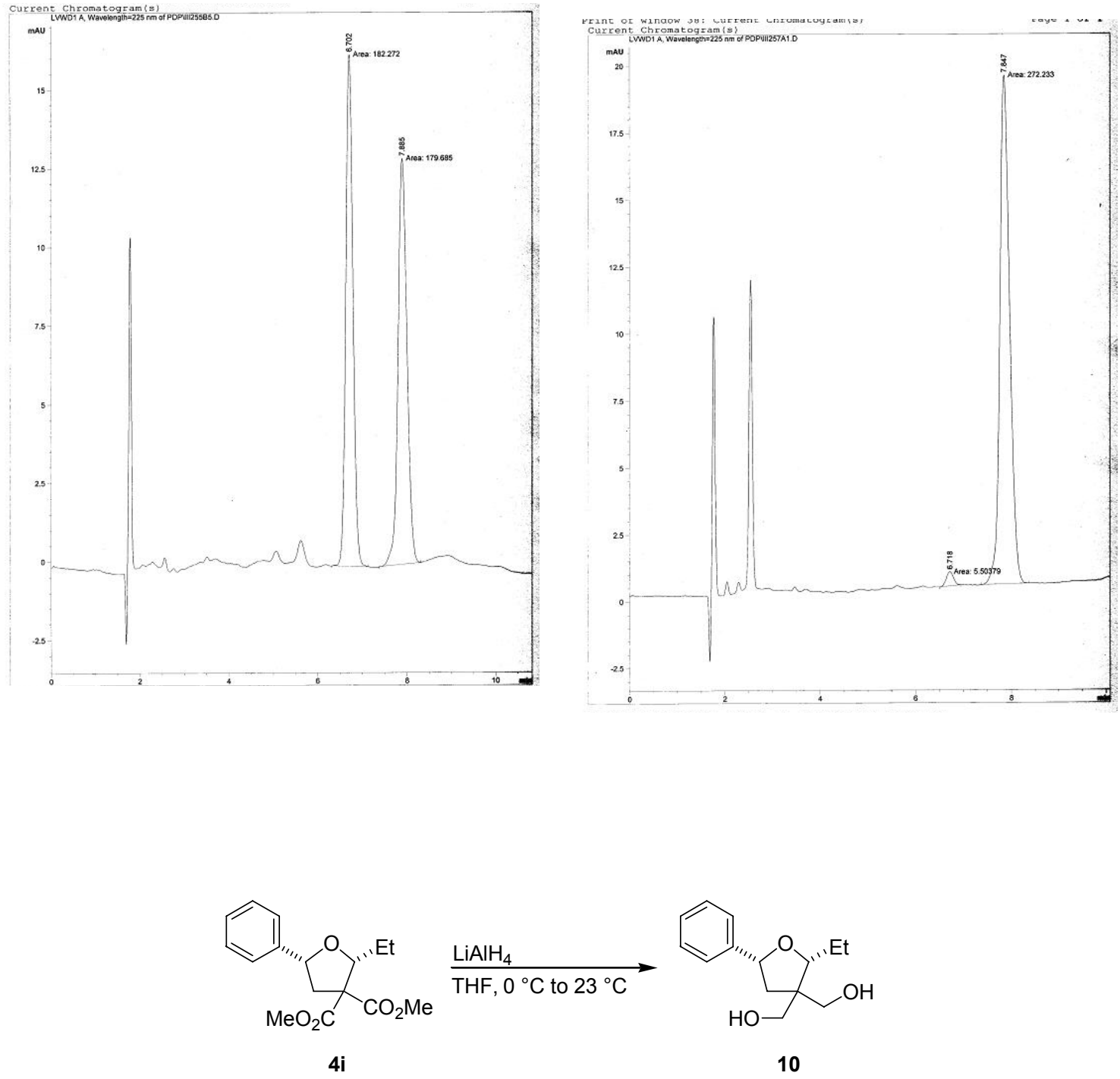

(2-Ethyl-3-hydroxymethyl-5-phenyl-tetrahydro-furan-3-yl)-methanol (10).

A stirring suspension of $\mathrm{LiAlH}_{4}\left(50.2 \mathrm{mg}, 1.32 \mathrm{mmol}, 5.0\right.$ equiv) in $1.5 \mathrm{~mL}$ of THF at $0{ }^{\circ} \mathrm{C}$ 
was treated with a solution of $4 \mathbf{i}(77.3 \mathrm{mg}, 0.264 \mathrm{mmol}, 1.0$ equiv) in $1.5 \mathrm{~mL}$ of THF via syringe. After addition the reaction was allowed to warm to room temperature with stirring. After $1.5 \mathrm{~h}$ the reaction was quenched with $50 \mu \mathrm{L} \mathrm{H}_{2} \mathrm{O}, 50 \mu \mathrm{L}$ of a $15 \% \mathrm{NaOH}$ (aq.) solution, and $150 \mu \mathrm{L}$ of $\mathrm{H}_{2} \mathrm{O}$. The solution was filtered through a Büchner funnel and the filter cake was washed with several portions of $\mathrm{Et}_{2} \mathrm{O}$. The filtrate was dried over $\mathrm{MgSO}_{4}$, filtered, and concentrated by rotary evaporation affording a white solid. The crude product was purified by flash chromatography with $60 \%$ EtOAc/petroleum ether to afford $54 \mathrm{mg}(87 \%)$ of the product as a white solid in $96 \%$ ee as determined by chiral SFC analysis (Chiralpak AD, 10.0\% MeOH, $2.0 \mathrm{~mL} / \mathrm{min}, 200 \mathrm{psi}, 40{ }^{\circ} \mathrm{C}, 225 \mathrm{~nm}, t_{\mathrm{r}-\text { major }}$ $\left.9.2 \mathrm{~min}, t_{\mathrm{r}-\mathrm{minor}} 6.9 \mathrm{~min}\right)$.

Analytical data for 10: $[\alpha]_{\mathrm{D}}{ }^{27}+69.0\left(\mathrm{c}=1.03, \mathrm{CH}_{2} \mathrm{Cl}_{2}\right) ; \mathrm{mp}=82-84{ }^{\circ} \mathrm{C}$; IR (Nujol mull, $\mathrm{cm}^{-1}$ ) 3298, 1128, 1059, 1024, 987, 957, 762, 700; ${ }^{1} \mathbf{H}$ NMR (400 MHz, $\left.\mathrm{CDCl}_{3}\right) \delta 7.40-7.22(\mathrm{~m}, 5 \mathrm{H}), 4.88(\mathrm{dd}, J=9.2,6.8 \mathrm{~Hz}, 1 \mathrm{H}), 3.89(\mathrm{~d}, J=10.8 \mathrm{~Hz}, 1 \mathrm{H})$, $3.84(\mathrm{~s}, 2 \mathrm{H}), 3.72(\mathrm{~d}, J=10.4 \mathrm{~Hz}, 1 \mathrm{H}), 3.66(\mathrm{dd}, J=10.0,3.2 \mathrm{~Hz}, 1 \mathrm{H}), 2.29$ (dd, $J=$ 13.2, 7.2 Hz, 1H), 2.08 (s, 2H), $1.76(\mathrm{dd}, J=12.8,9.2 \mathrm{~Hz}, 1 \mathrm{H}), 1.78-1.54(\mathrm{~m}, 2 \mathrm{H}), 1.10$ $(\mathrm{t}, J=7.6 \mathrm{~Hz}, 3 \mathrm{H}) ;{ }^{13} \mathbf{C}$ NMR $\left(75 \mathrm{MHz}, \mathrm{CDCl}_{3}\right) \delta 142.5,128.5,127.5,125.9,85.7,79.0$, 67.8, 66.0, 50.9, 41.1, 24.1, 12.0; TLC (40\% EtOAc/petroleum ether) $\mathrm{R}_{f}$ 0.07; Anal. Calcd. for $\mathrm{C}_{14} \mathrm{H}_{20} \mathrm{O}_{3}$ : C, 71.16; H, 8.53. Found: C, 71.21; H, 8.62.

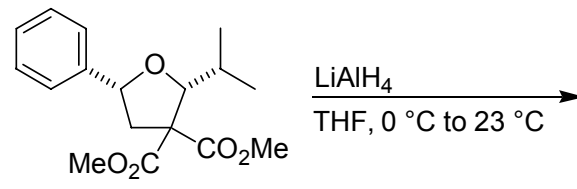

$4 \mathbf{j}$

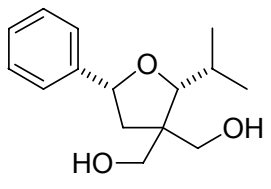

11

(3-Hydroxymethyl-2-isopropyl-5-phenyl-tetrahydro-furan-3-yl)-methanol (11). A stirring suspension of $\mathrm{LiAlH}_{4}\left(42.4 \mathrm{mg}, 1.12 \mathrm{mmol}, 5.0\right.$ equiv) in $1.5 \mathrm{~mL}$ of THF at $0{ }^{\circ} \mathrm{C}$ was treated with a solution of $\mathbf{4 j}(68.5 \mathrm{mg}, 0.224 \mathrm{mmol}, 1.0$ equiv) in $1.5 \mathrm{~mL}$ of THF via syringe. After addition the reaction was allowed to warm to room temperature with stirring. After $1.5 \mathrm{~h}$ the reaction was quenched with $40 \mu \mathrm{L} \mathrm{H}_{2} \mathrm{O}, 40 \mu \mathrm{L}$ of a $15 \% \mathrm{NaOH}$ (aq.) solution, and $120 \mu \mathrm{L}$ of $\mathrm{H}_{2} \mathrm{O}$. The solution was filtered through a Büchner funnel and the filter cake was washed with several portions of $\mathrm{Et}_{2} \mathrm{O}$. The filtrate was dried over $\mathrm{MgSO}_{4}$, filtered, and concentrated by rotary evaporation affording a white solid. The crude product was purified by flash chromatography with $50 \%$ EtOAc/petroleum ether to afford $51 \mathrm{mg}(91 \%)$ of the product as a white solid in $95 \%$ ee as determined by chiral SFC analysis (Chiralpak AD, 10.0\% MeOH, $2.0 \mathrm{~mL} / \mathrm{min}, 200 \mathrm{psi}, 40{ }^{\circ} \mathrm{C}, 225 \mathrm{~nm}, t_{\mathrm{r}-\mathrm{major}}$ $\left.7.9 \mathrm{~min}, t_{\mathrm{r}-\mathrm{minor}} 6.7 \mathrm{~min}\right)$.

Analytical data for 11: $[\alpha]_{\mathrm{D}}{ }^{26}+54.3\left(\mathrm{c}=1.03, \mathrm{CH}_{2} \mathrm{Cl}_{2}\right) ; \mathrm{mp}=78.5-80{ }^{\circ} \mathrm{C}$; IR (Nujol mull, $\mathrm{cm}^{-1}$ ) 3255, 1134, 1097, 1063, 1045, 1012, 760, 702; ${ }^{1} \mathbf{H}$ NMR (300 MHz, $\left.\mathrm{CDCl}_{3}\right) \delta 7.44-7.20(\mathrm{~m}, 5 \mathrm{H}), 4.79(\mathrm{dd}, J=7.5,7.5 \mathrm{~Hz}, 1 \mathrm{H}), 3.88(\mathrm{~d}, J=10.5 \mathrm{~Hz}, 1 \mathrm{H})$, $3.82(\mathrm{~d}, J=10.8 \mathrm{~Hz}, 1 \mathrm{H}), 3.62(\mathrm{~d}, J=10.2 \mathrm{~Hz}, 1 \mathrm{H}), 3.60(\mathrm{~d}, J=10.5 \mathrm{~Hz}, 1 \mathrm{H}), 3.38$ (s, $2 \mathrm{H}), 3.22(\mathrm{~d}, J=7.8 \mathrm{~Hz}, 1 \mathrm{H}), 2.38(\mathrm{dd}, J=12.9,7.2 \mathrm{~Hz}, 1 \mathrm{H}), 1.96-1.75(\mathrm{~m}, 2 \mathrm{H}), 1.03$ (d, $J=6.6 \mathrm{~Hz}, 3 \mathrm{H}), 0.95(\mathrm{~d}, J=6.6 \mathrm{~Hz}, 3 \mathrm{H}) ;{ }^{13} \mathbf{C ~ N M R}\left(75 \mathrm{MHz}, \mathrm{CDCl}_{3}\right) \delta 142.4,128.4$, 
127.4, 125.8, 89.0, 78.2, 69.0, 66.6, 51.1, 41.8, 28.7, 20.8 (two overlapping resonances); TLC (60\% EtOAc/petroleum ether) $\mathrm{R}_{f}$ 0.37; Anal. Calcd. for $\mathrm{C}_{15} \mathrm{H}_{22} \mathrm{O}_{3}: \mathrm{C}, 71.97 ; \mathrm{H}$, 8.86. Found: C, 71.96; H, 8.93.
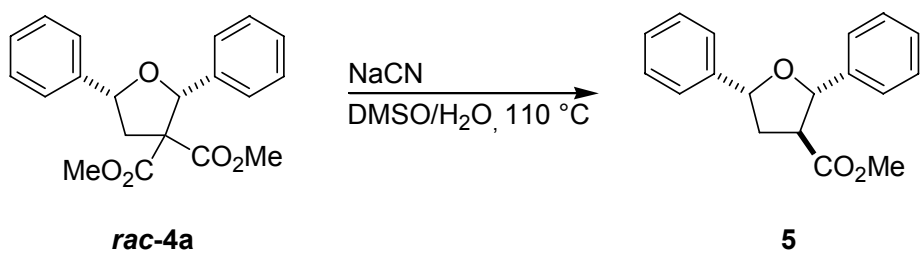

5

2,5-Diphenyl-tetrahydro-furan-3-carboxylic acid methyl ester (5). A 10-mL round bottomed flask was charged with rac-4a $(80.0 \mathrm{mg}, 0.235 \mathrm{mmol}, 1.0$ equiv), $\mathrm{NaCN}(21.0$ mg, $0.428 \mathrm{mmol}, 1.8$ equiv), $2 \mathrm{~mL}$ of DMSO, and $\mathrm{H}_{2} \mathrm{O}$ (20.0 mg, $1.11 \mathrm{mmol}, 4.7$ equiv). The flask was affixed with a reflux condenser and the reaction was heated to $110{ }^{\circ} \mathrm{C}$ with stirring. After $20 \mathrm{~h}$, the reaction was cooled to room temperature and partitioned between $20 \mathrm{~mL}$ of $\mathrm{Et}_{2} \mathrm{O}$ and $20 \mathrm{~mL}$ of water. After separation the aqueous layer was extracted with three $20 \mathrm{~mL}$ portions of $\mathrm{Et}_{2} \mathrm{O}$. The combined organic extracts were washed with three $20 \mathrm{~mL}$ portions of water and $20 \mathrm{~mL}$ of brine, dried over $\mathrm{MgSO}_{4}$, filtered and concentrated by rotary evaporation affording a colorless oil. ${ }^{1} \mathrm{H}$ NMR analysis of the unpurified product ( $\delta 2.72$ vs. $\delta 5.28$ ) gave the diastereomeric ratio: 6.7:1. The isomers were separated and purified by flash chromatography with $5 \%$ EtOAc/petroleum ether to afford $53 \mathrm{mg}(80 \%)$ of the products as a colorless oil (major diastereomer) and a white solid (minor diastereomer).

Analytical data for 5: When performed with $97 \%$ ee $4 \mathbf{a} ;[\alpha]_{\mathrm{D}}{ }^{26}+50.8(\mathrm{c}=1.04$, $\mathrm{CH}_{2} \mathrm{Cl}_{2}$ ); IR (thin film, $\mathrm{cm}^{-1}$ ) 3089, 3064, 3032, 2951, 1736, 1605, 1497, 1452, 1435, 1358, 1308, 1263, 1196, 1171, 1086, 1059, 1026, 756, 698; ${ }^{\mathbf{1}} \mathbf{H}$ NMR (400 MHz, CDCl $\left.{ }_{3}\right)$ $\delta 7.55-7.22(\mathrm{~m}, 10 \mathrm{H}), 5.20(\mathrm{~d}, J=7.2 \mathrm{~Hz}, 1 \mathrm{H}), 5.16(\mathrm{dd}, J=7.6,7.6 \mathrm{~Hz}, 1 \mathrm{H}), 3.75(\mathrm{~s}$, $3 \mathrm{H}$ ), 3.13 (ddd, $J=10.4,7.2,5.2 \mathrm{~Hz}, 1 \mathrm{H}$ ), 2.72 (ddd, $J=12.8,6.8,5.2 \mathrm{~Hz}, 1 \mathrm{H}$ ), 2.23 (ddd, $J=12.8,9.2,9.2 \mathrm{~Hz}, 1 \mathrm{H}) ;{ }^{13} \mathbf{C}$ NMR $\left(100 \mathrm{MHz}, \mathrm{CDCl}_{3}\right) \delta 174.1,141.5,141.1$, 128.6, 128.0, 127.8, 126.2, 126.1, 83.8, 80.9, 52.3, 52.2, 38.9 (two overlapping $\mathrm{sp}^{2}$ resonances); TLC (5\% EtOAc/petroleum ether) $\mathrm{R}_{f} 0.18$; Anal. Calcd. for $\mathrm{C}_{18} \mathrm{H}_{18} \mathrm{O}_{3}$ : C, 76.57; H, 6.43. Found: C, 76.39; H, 6.36. 


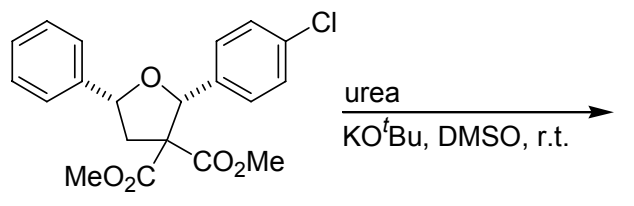

4b

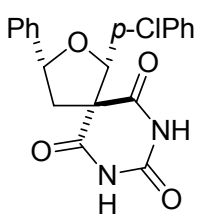

12

(1R,3R)-1-(4-Chloro-phenyl)-3-phenyl-2-oxa-7,9-diaza-spiro[4.5]decane-6,8,10-

trione (12). A solution of $\mathbf{4 b}(96 \%$ ee) $(114.3 \mathrm{mg}, 0.305 \mathrm{mmol}, 1.0$ equiv) in $0.6 \mathrm{~mL}$ DMSO was treated with urea $\left(108.0 \mathrm{mg}, 1.80 \mathrm{mmol}, 5.9\right.$ equiv) and $\mathrm{KO}^{t} \mathrm{Bu}(75.3 \mathrm{mg}$, $0.671 \mathrm{mmol}, 2.2$ equiv). After stirring for $1 \mathrm{~h}$, the reaction was diluted with $15 \mathrm{~mL}$ of EtOAc and washed with $20 \mathrm{~mL}$ of a $0.1 \mathrm{~N} \mathrm{HCl}$ (aq.) solution. The aqueous phase was extracted with three $20 \mathrm{~mL}$ portions of EtOAc. The combined organics were washed with two $20 \mathrm{~mL}$ portions of water and $25 \mathrm{~mL}$ of brine, dried over $\mathrm{MgSO}_{4}$, filtered, and concentrated by rotary evaporation affording a white solid. The crude product was purified by flash chromatography with a $30 \%$ to $40 \%$ EtOAc/petroluem ether gradient to afford $58 \mathrm{mg}(51 \%)$ of the product as a white solid. A portion of this material was dissolved in a small amount of THF and recrystallized (for x-ray analysis) by slow diffusion of petroleum ether vapor into the solution.

Analytical data for 12: $[\alpha]_{\mathrm{D}}{ }^{26}+71.0(\mathrm{c}=1.00, \mathrm{THF}) ; \mathrm{mp}=263-265{ }^{\circ} \mathrm{C}$; IR (Nujol mull, $\mathrm{cm}^{-1}$ ) 3236, 1759, 1728, 1705, 1194, 843, 762; ${ }^{\mathbf{H}} \mathbf{H}$ NMR (300 MHz, CDCl 3 ) $\delta 11.43(\mathrm{~s}, 1 \mathrm{H}), 10.78(\mathrm{~s}, 1 \mathrm{H}), 7.64(\mathrm{~d}, J=6.9 \mathrm{~Hz}, 2 \mathrm{H}), 7.50-7.25(\mathrm{~m}, 7 \mathrm{H}), 5.12(\mathrm{dd}, J=$ 9.0, $9.0 \mathrm{~Hz}, 1 \mathrm{H}), 5.07(\mathrm{~s}, 1 \mathrm{H}), 2.93$ (dd, $J=12.9,7.2 \mathrm{~Hz}, 1 \mathrm{H}), 2.55$ (dd, $J=12.9,9.6 \mathrm{~Hz}$, $1 \mathrm{H}) ;{ }^{13} \mathrm{C}$ NMR $\left(100 \mathrm{MHz}, \mathrm{CDCl}_{3}\right) \delta 171.8,170.3,150.1,140.5,134.5,133.4,128.4$, 128.3, 128.1, 128.0, 126.7, 89.3, 81.1, 63.7, 42.8; TLC (40\% EtOAc/petroleum ether) $\mathrm{R}_{f}$ 0.30; Anal. Calcd. for $\mathrm{C}_{19} \mathrm{H}_{15} \mathrm{ClN}_{2} \mathrm{O}_{4}$ : C, 61.55; H, 4.08; N, 7.56. Found: C, 61.58; H, $4.11 ; \mathrm{N}, 7.49$. 


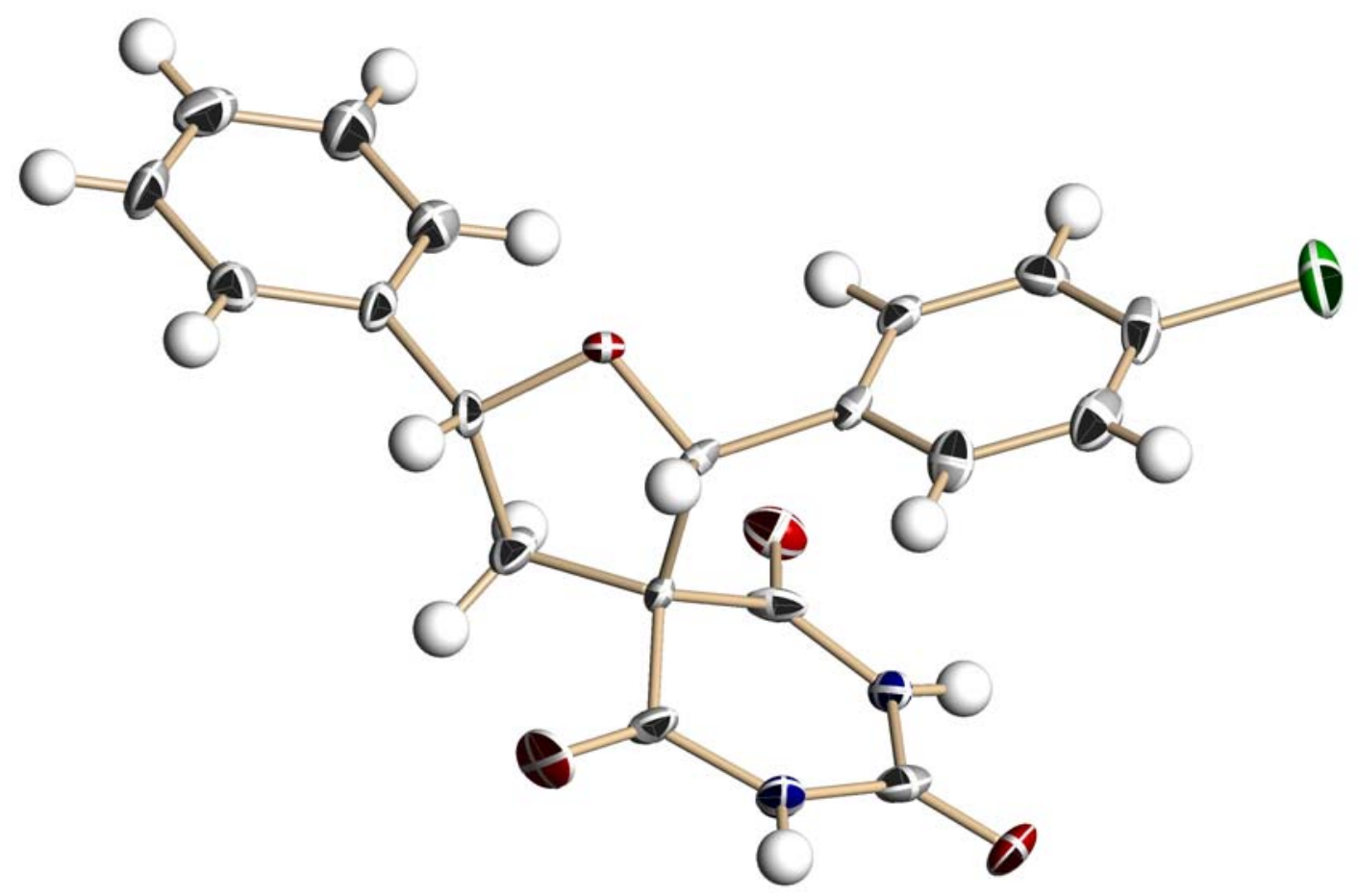

Stereochemical Proof for 7

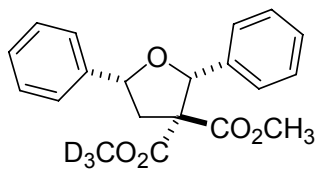

7

The stereochemistry of 7 is assigned based of the fact that every tetrahydrofuran derivative with a 2-aryl group shows one downfield resonance $(\sim 3.7 \mathrm{ppm})$ and one upfield resonance $(\sim 3.2 \mathrm{ppm})$ for the two diastereotopic carbomethoxy groups in the ${ }^{1} \mathrm{H}$ NMR. The downfield resonance arises from the ester group trans to the aryl ring (see spectra of 5). The upfield resonance arises from the ester group cis to the aryl ring, presumably do to anisotropy.

\section{References}

(1) Davies, H. M. L.; Bruzinski, P.; Hutcheson, D. K.; Kong, N.; Fall, M. J. J. Am. Chem. Soc. 1996, 118, 6897-6907.

(2) Corey, E. J.; Gant, T. G. Tetrahedron Lett. 1994, 35, 5373-5376.

(3) Fraser, W.; Suckling, C. J.; Wood, H. C. S. J. Chem. Soc. Perkin Trans. 1. 1990, 3137-3144. 


\section{Appendix. NMR spectra}

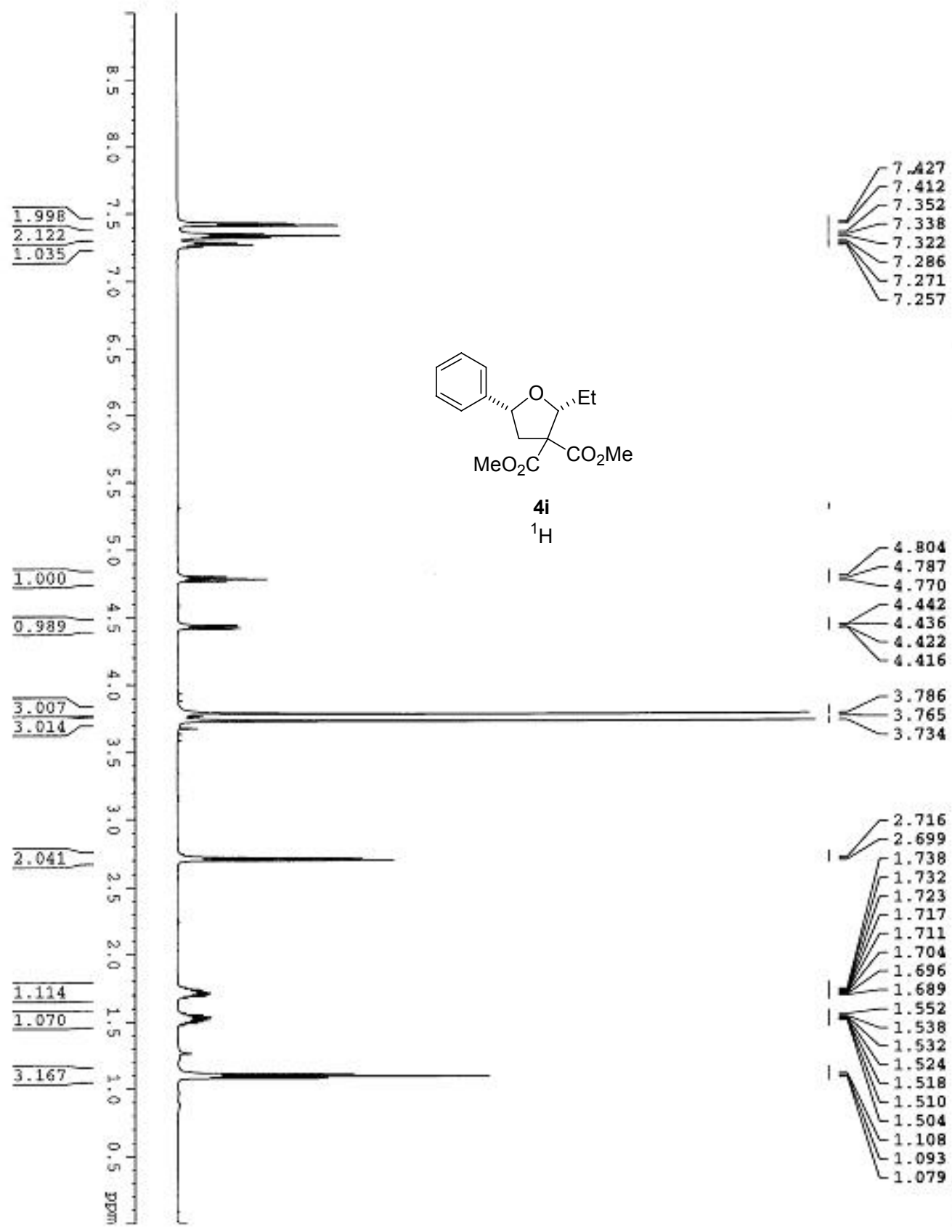




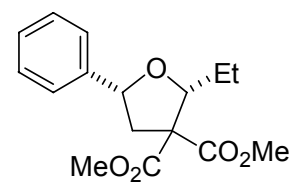

$4 i$

noesy

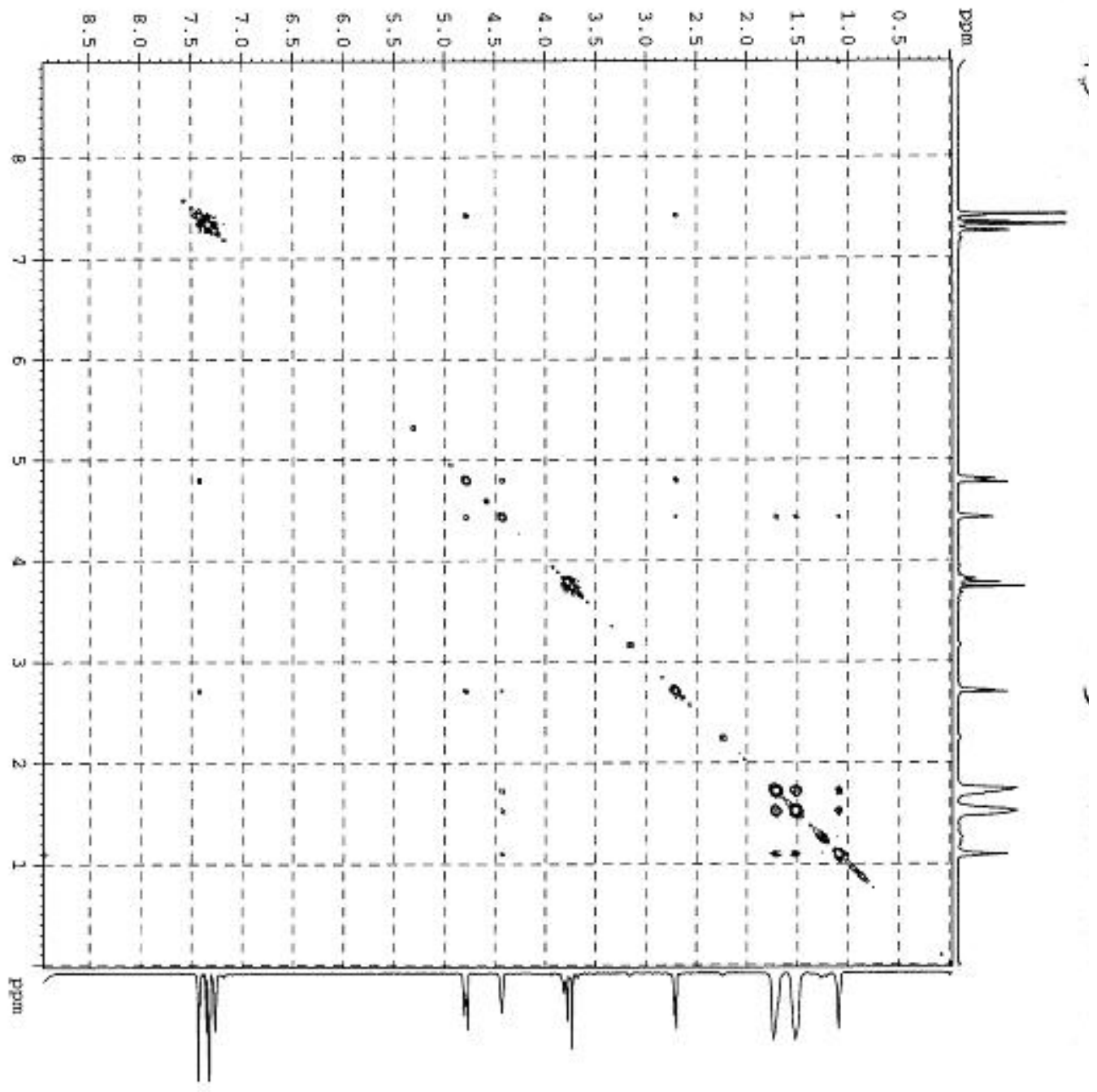




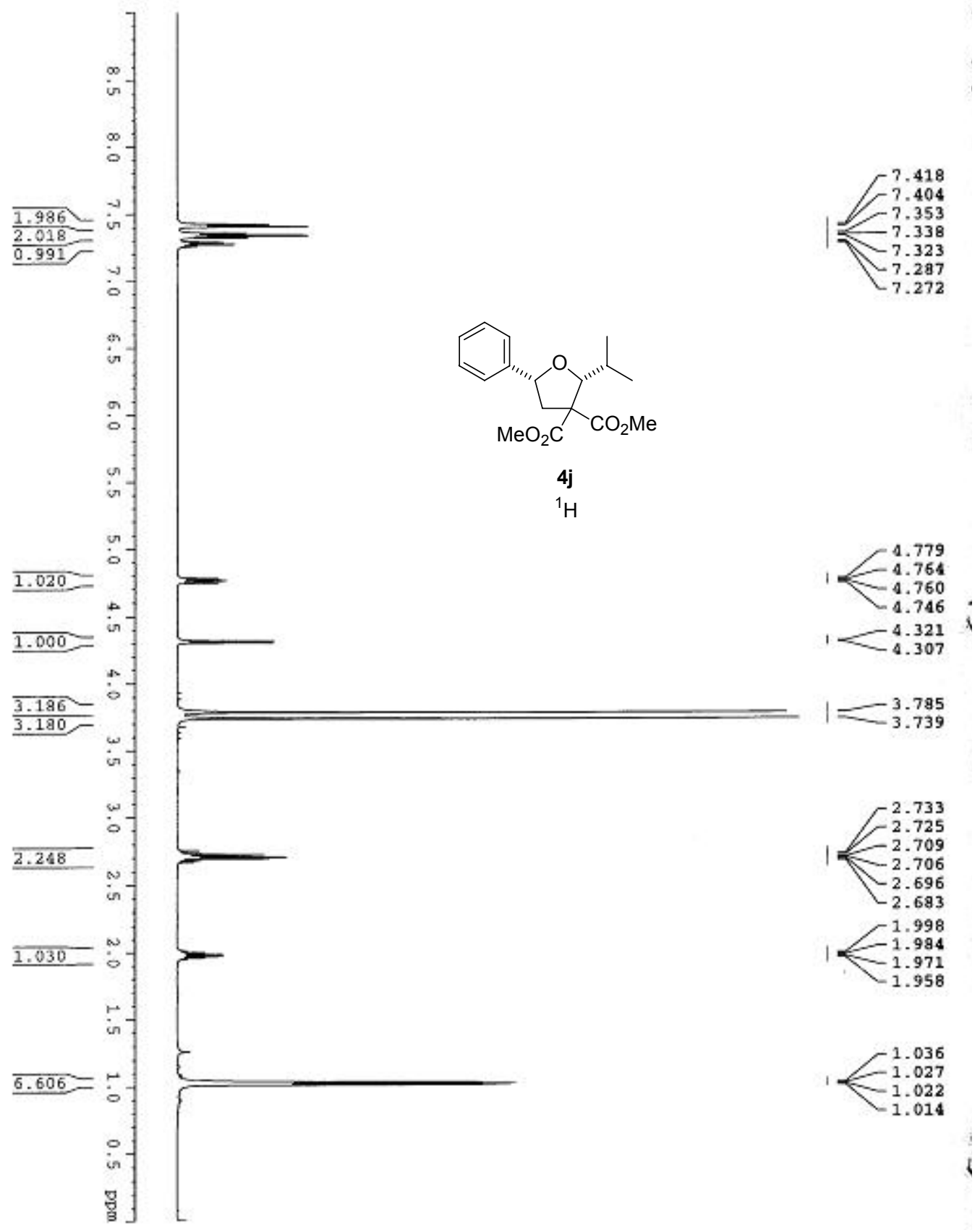




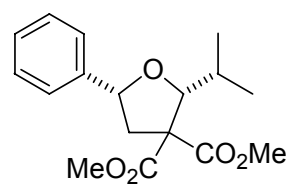

4j

noesy

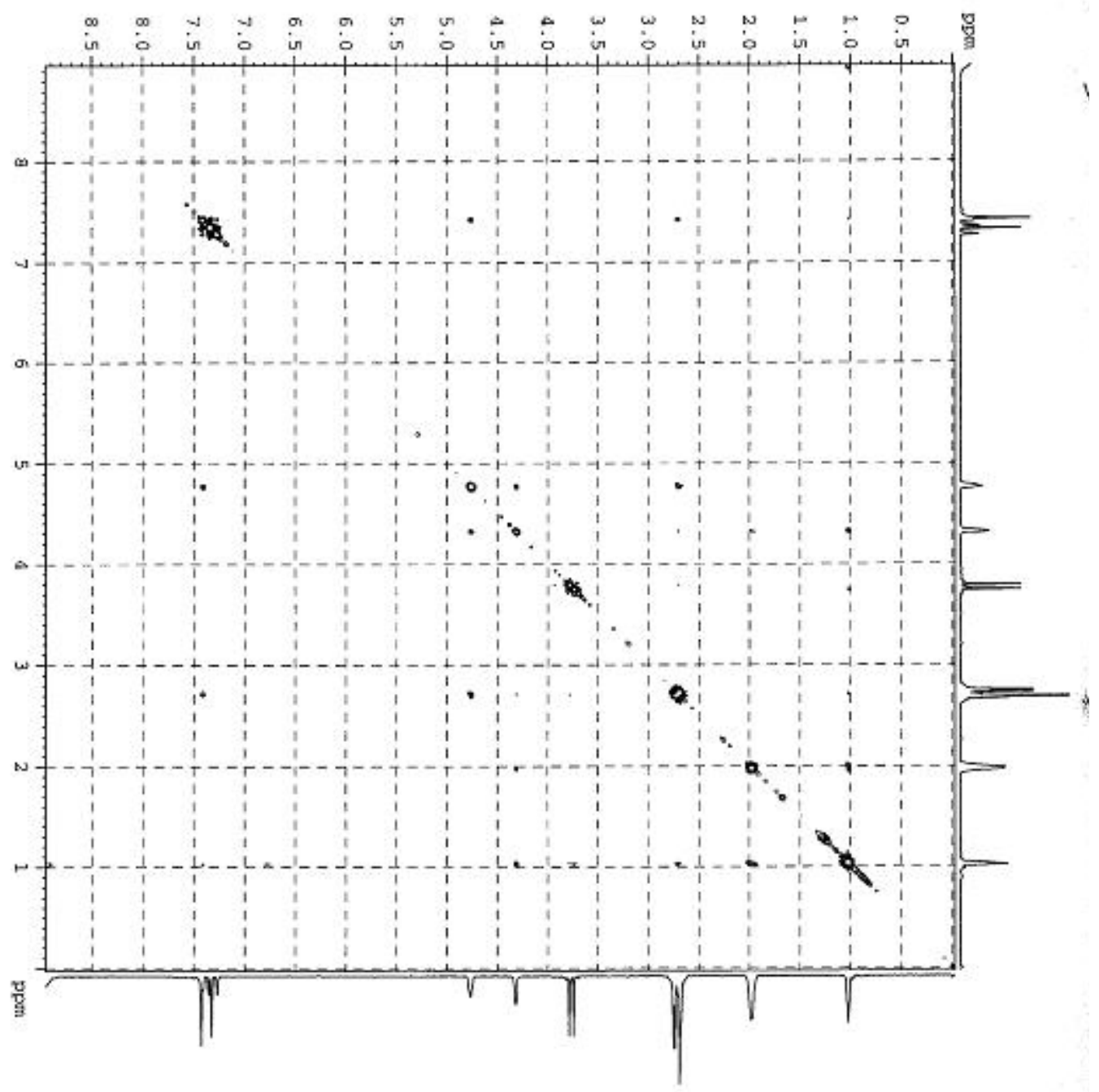




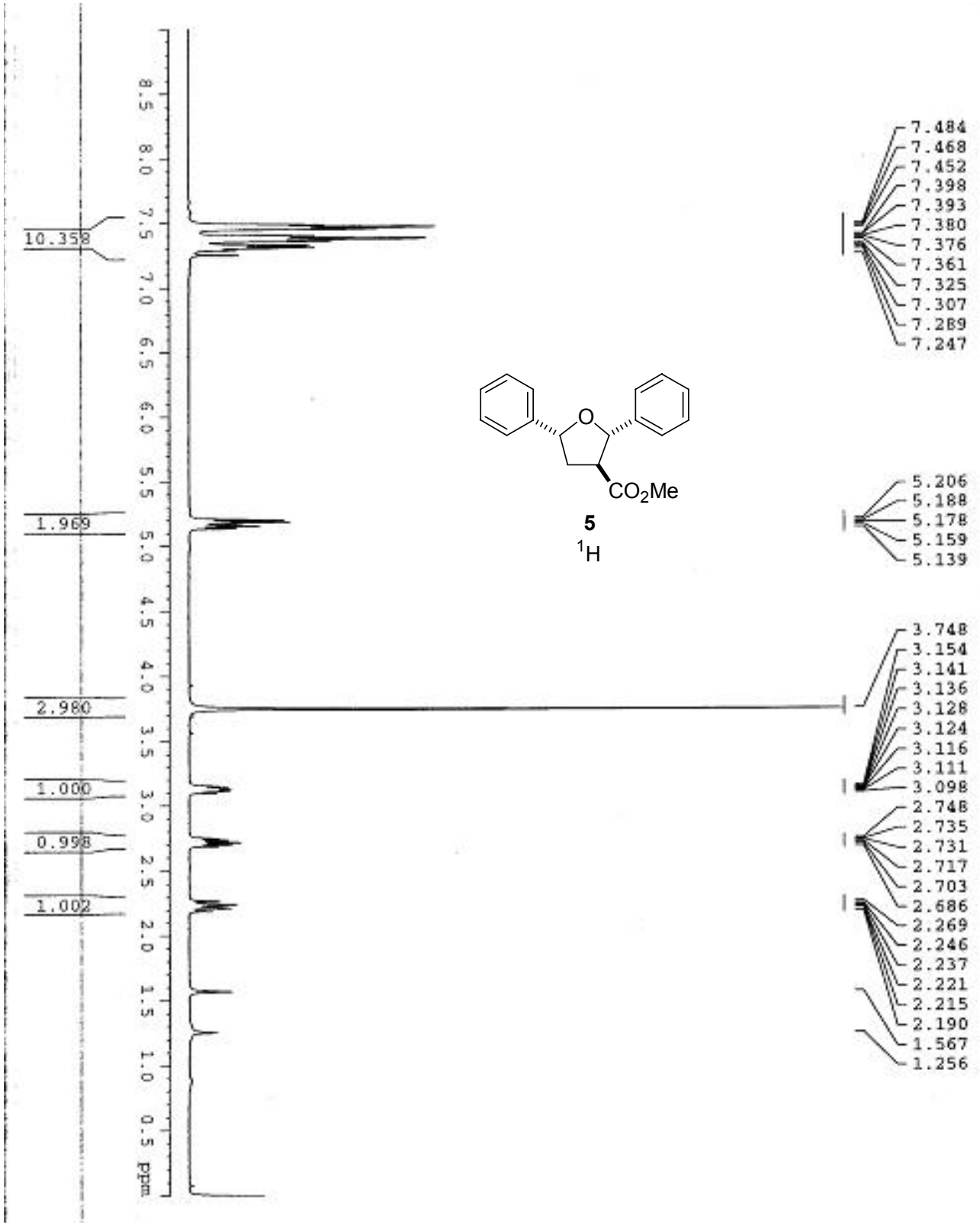



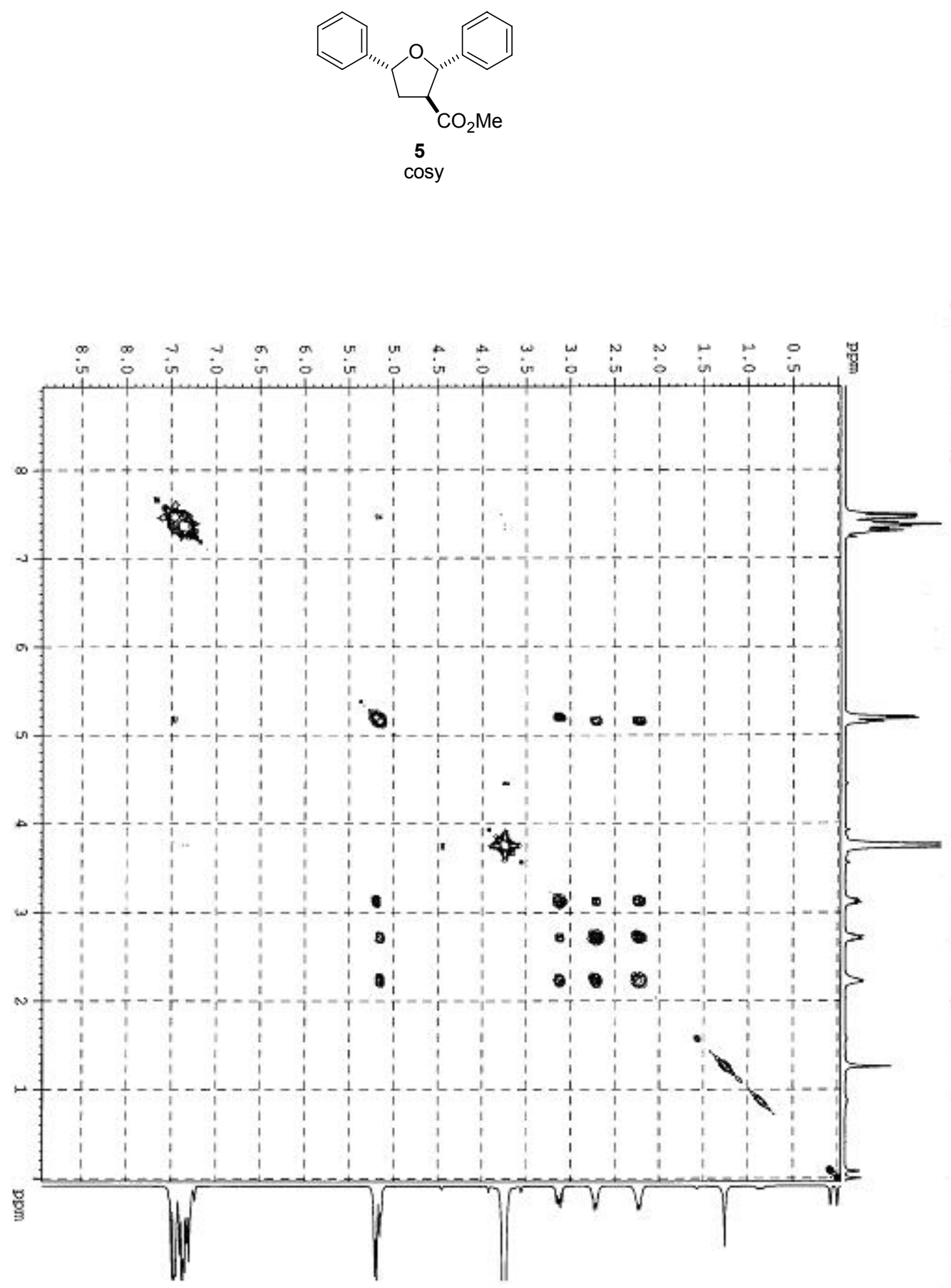

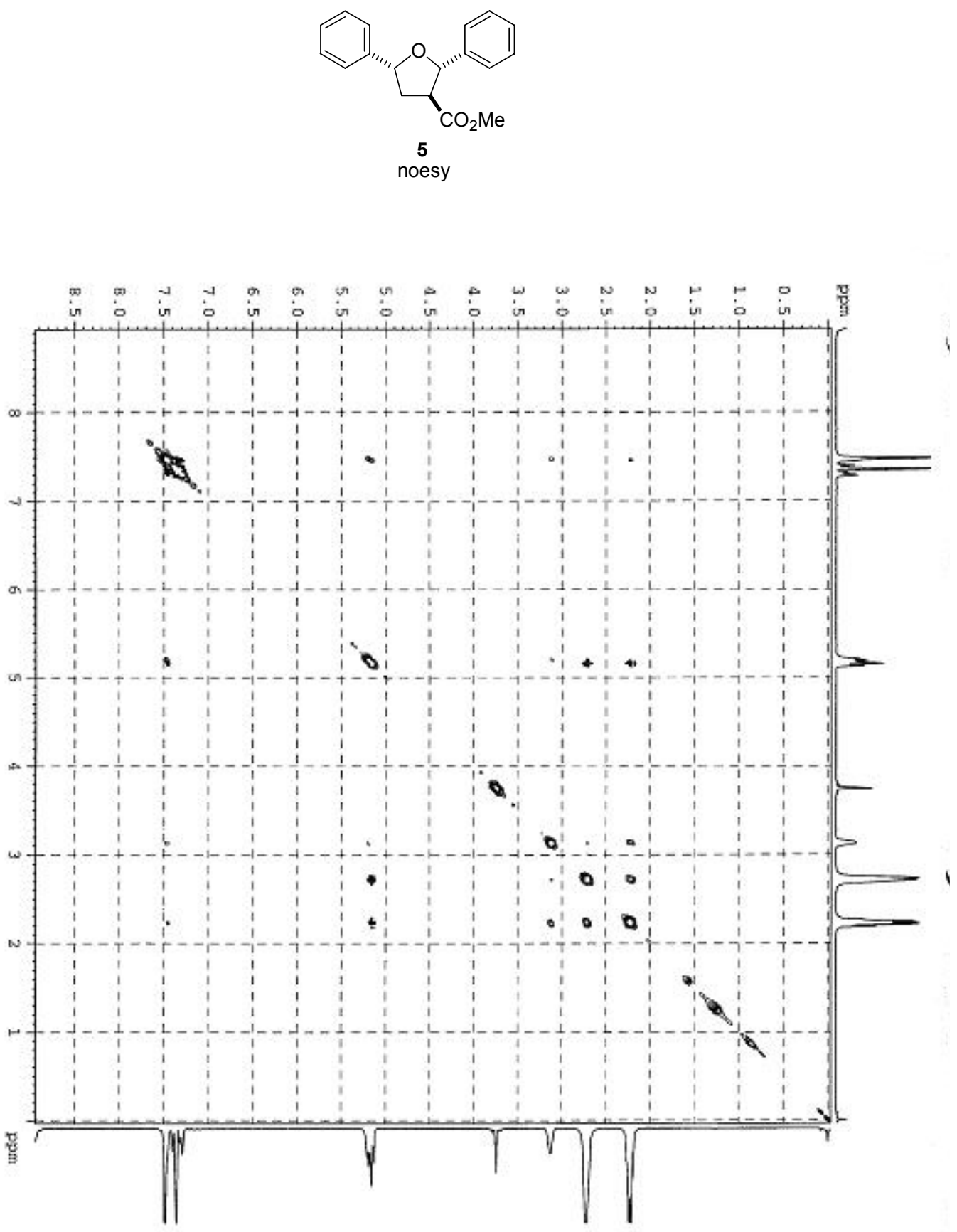


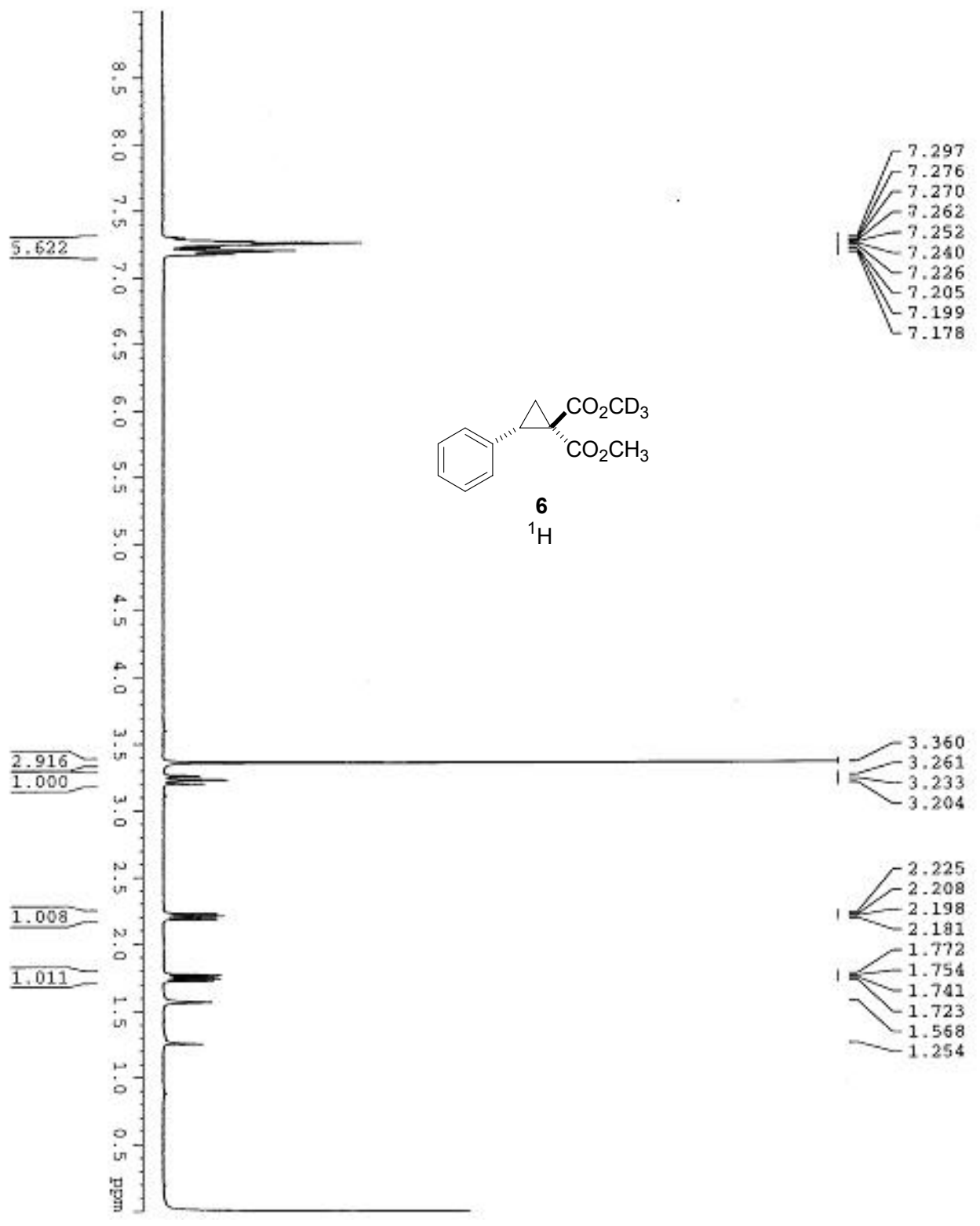




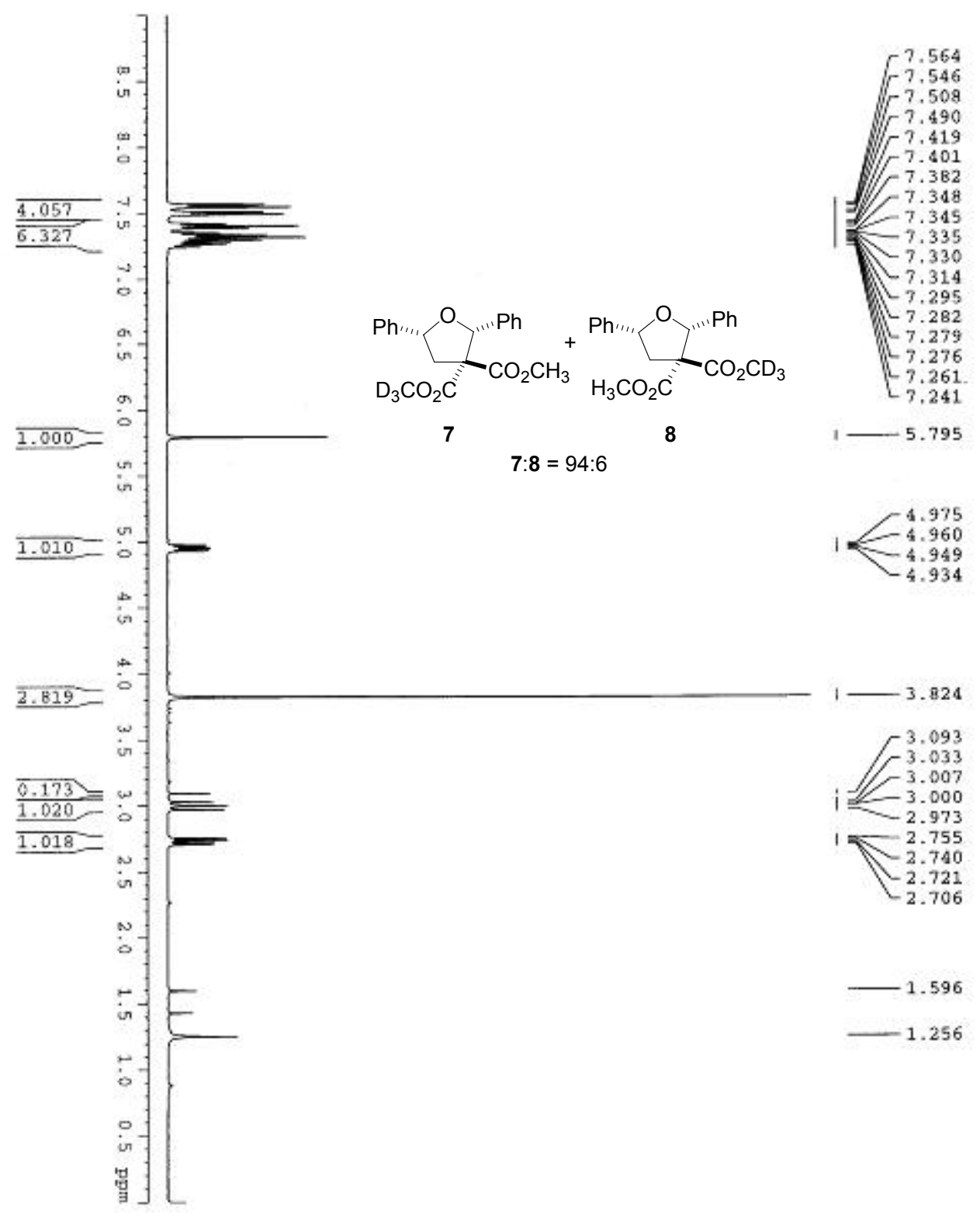

